\title{
SPECTROSCOPIC INFRARED EXTINCTION MAPPING AS A PROBE OF GRAIN GROWTH IN IRDCs
}

\author{
WangGi Lim ${ }^{1,2}$, Sean J. CareY ${ }^{2}$, and Jonathan C. TaN ${ }^{3}$ \\ ${ }^{1}$ Department of Astronomy, University of Florida, Gainesville, FL 32611, USA \\ ${ }^{2}$ Infrared Processing Analysis Center, California Institute of Technology, Pasadena, CA 91125, USA \\ ${ }^{3}$ Departments of Astronomy \& Physics, University of Florida, Gainesville, FL 32611, USA \\ Received 2015 August 10; accepted 2015 October 9; published 2015 November 13
}

\begin{abstract}
We present spectroscopic tests of MIR to FIR extinction laws in IRDC G028.36+00.07, a potential site of massive star and star cluster formation. Lim \& Tan developed methods of FIR extinction mapping of this source using Spitzer-MIPS $24 \mu \mathrm{m}$ and Herschel-PACS $70 \mu \mathrm{m}$ images, and by comparing to MIR Spitzer-IRAC 3-8 $\mu \mathrm{m}$ extinction maps, found tentative evidence for grain growth in the highest mass surface density regions. Here we present results of spectroscopic infrared extinction mapping using Spitzer-IRS (14-38 $\mu \mathrm{m})$ data of the same Infrared dark cloud (IRDC). These methods allow us to first measure the SED of the diffuse Galactic interstellar medium that is in the foreground of the IRDC. We then carry out our primary investigation of measuring the MIR to FIR opacity law and searching for potential variations as a function of mass surface density within the IRDC. We find relatively flat, featureless MIR-FIR opacity laws that lack the $\sim 12$ and $\sim 35 \mu$ m features associated with the thick water ice mantle models of Ossenkopf \& Henning. Their thin ice mantle models and the coagulating aggregate dust models of Ormel et al. are a generally better match to the observed opacity laws. We also find evidence for generally flatter MIR to FIR extinction laws as mass surface density increases, strengthening the evidence for grain and ice mantle growth in higher density regions.
\end{abstract}

Key words: dust, extinction - infrared: ISM - ISM: clouds - stars: formation

\section{INTRODUCTION}

Massive star and star cluster formation are key processes for understanding galaxy evolution, since massive stars are vital sources of feedback for regulating the interstellar medium (ISM) and further star formation activity, while star clusters are the basic building blocks of galactic stellar populations. Despite this importance, we understand massive star and star cluster formation only very poorly because the regions forming such objects are relatively rare and thus typically far away (at least a few kpcs for most Galactic sources) and also deeply embedded inside dense molecular clouds.

A better understanding of massive star and star cluster formation should result if we can find and characterize the initial conditions of these processes. Two key methods to achieve this involve studying the amount of IR extinction and sub-mm $/ \mathrm{mm}$ emission of dust grains in cold, dense regions of molecular clouds in order to measure mass surface densities and thus masses of cloud structures (see, e.g., Tan et al. 2014 for a review, hereafter T14). To do this accurately, one needs to know the opacities and emissivities of the dust grains, including potential systematic evolution in these properties. This is the goal of our study. A better understanding of dust properties will also be important for modeling heating and cooling in the clouds, astrochemistry, and the ionization fraction, which affects dynamics by enabling coupling of the mostly neutral gas to magnetic fields.

The NIR to FIR properties of dust extinction (from 1 to $25 \mu \mathrm{m}$ ) have been measured in the Lupus molecular cloud using background stars by Boogert et al. (2013). They found evidence for water ice (presumably forming on dust grains) on sightlines with $A_{V} \gtrsim 2$ mag and that with increasing $A_{K}$ the $>5$ $\mu \mathrm{m}$ continuum extinction increases relative to $A_{K}$. The most extincted line of sight that they probed had $A_{K}=2.46 \mathrm{mag}$ $\left(A_{V} \sim 20 \mathrm{mag}\right)$. These are relatively low mass surface density conditions compared to the clouds that are thought to form massive stars and star clusters (T14). Lutz et al. (1996) studied the MIR extinction (MIREX) law (from 2.5 to $9 \mu \mathrm{m}$ ) toward the Galactic Center using hydrogen recombination lines. They found a relatively flat extinction law, e.g., compared to the bare grain models of Draine (1989). This sightline has $A_{V} \sim 30 \mathrm{mag}$, but is essentially probing diffuse ISM conditions that are not likely to be close to initial conditions for star formation. McClure (2009) studied 5-20 $\mu \mathrm{m}$ dust extinction properties on sightlines through molecular clouds with $0.3 \leqslant A_{K}<7$ towards background stars, finding systematic evolution with increasing $A_{K}$ that was attributed to ice-mantle mediated grain coagulation (see also Flaherty et al. 2007; Chapman et al. 2009; c.f., Ascenso et al. 2013, who did not find evidence for extinction law variations up to $A_{V} \sim 50 \mathrm{mag}$ ).

Infrared dark clouds (IRDCs) (e.g., Perault et al. 1996; Carey et al. 1998; Ormel et al. 2005; Rathborne et al. 2005; T14) cast shadows at MIR wavelengths, $\sim 10 \mu \mathrm{m}$. They are occasionally opaque at far-infrared wavelengths, up to $\sim 100 \mu \mathrm{m}$, against the Galactic diffuse background emission (e.g., Lim \& Tan 2014, hereafter LT14) due to their very high mass surface densities $\sim 1 \mathrm{~g} \mathrm{~cm}^{-2}\left(A_{V} \sim 200 \mathrm{mag}\right)$ and cold $\lesssim 15 \mathrm{~K}$ temperatures. Being massive clouds with such physical conditions, IRDCs are likely to be representative of the initial conditions for massive star and star cluster formation (T14).

Butler \& Tan (2009, 2012; hereafter BT09, BT12) studied mass surface densities, $\Sigma$, of 42 starless cores inside 10 IRDCs via MIREX mapping by utilizing Spitzer-IRAC $8 \mu \mathrm{m}$ data of the GLIMPSE survey (Churchwell et al. 2009). Butler et al. (2014, hereafter BTK14) used deeper Spitzer-IRAC $8 \mu \mathrm{m}$ images toward one of the 10 BT09 IRDCs (IRDC G028.37 +00.07 , also referred to as IRDC C), including NIR extinction offset corrections of Kainulainen \& Tan (2013), to produce a higher dynamic range extinction map.

The advantages of such MIREX mapping are high angular resolution $\left(2^{\prime \prime}\right)$, a near-fully sampled image (except in regions 
of bright MIR emission) and an independence to dust temperature (except, again, in very warm regions that are sources of MIR emission). A major uncertainty is the need to estimate the background intensity by interpolation from regions around the cloud. The background intensity can also have significant small scale fluctuations, which, however, can be assessed from the off-cloud regions, but which are an inherent random uncertainty. Another major issue is the need to estimate the level of the MIR foreground intensity from the diffuse ISM, since IRDCs are typically at several kpc distances. The key method to estimate the foreground is to measure it empirically in spatially independent "saturated" regions of the IRDC where essentially negligible background light makes it through the cloud (as long as such regions exist), and then assume it is spatially invariant across the IRDC or some local region (BT12). The accuracy of measuring the saturated intensity level depends on the photometric precision of the image and sets a maximum $\Sigma_{\text {sat }}$ that can be probed in the map.

MIREX mapping does require knowing the $\sim 8 \mu \mathrm{m}$ opacity per unit mass: different dust models, such as the thin and thick Ossenkopf \& Henning (1994, hereafter OH94) moderately coagulated models or the Weingartner \& Draine (2001) bare grain models, show only modest variation at the level of $\sim 30 \%$. Then a (refractory)-dust to gas mass ratio also needs to be assumed (1:142) is our adopted fiducial value (Draine 2011; note BT09 and BT12 assumed 1:156). Overall, BT09, BT12 and BTK14 adopted the OH94 thin ice mantle model $\left(10^{5}\right.$ years of coagulation at density $\sim 10^{6} \mathrm{~cm}^{-3}$ ) as a fiducial, which has $\kappa_{8 \mu \mathrm{m}}$ (averaged over the IRAC band) $\simeq 7.5 \mathrm{~cm}^{2} \mathrm{~g}^{-1}$. With these methods, BT12 could trace details of highly extincted regions up to, $\Sigma_{\text {sat }} \sim 0.5 \mathrm{~g} \mathrm{~cm}^{-2}$, i.e., $A_{V} \sim 100 \mathrm{mag}$. BTK14 could reach somewhat higher $\Sigma_{\text {sat }} \sim 1 \mathrm{~g} \mathrm{~cm}^{-2}$, i.e., $A_{V} \sim 200 \mathrm{mag}$.

LT14 extended MIREX methods to the FIR regime by using Spitzer-MIPS $24 \mu \mathrm{m}$ MIPSGAL data (Carey et al. 2009) and Herschel-PACS $70 \mu \mathrm{m}$ archival data (proposal IDs: KPGTokrause-1 \& KPOT-smolinar-1) of IRDC G028.37+00.07. From studying $\sim 1^{\prime}$ regions around three dense, locally saturated cores (the white circles in Figure 1), LT14 found tentative evidence of $\kappa(\lambda)$ variation with $\Sigma$ in the sense that the relative extinction curves appear to flatten as $\Sigma$ increases. Such behavior is consistent with an evolution from the OH94 thin ice mantle model to the thick ice mantle model, in which there is a growth of the volume of the ice cover from $0.5 \times$ that of the refractory component to $4.5 \times$. Some degree of flattening could also result from coagulation of the grains (Cardelli et al. 1989; Weingartner \& Draine 2001; Ormel et al. 2011, hereafter O11).

In this paper, we present a new spectroscopic IR extinction (SIREX) mapping method by using Spitzer-IRS mid-infrared spectroscopic data that covers a part of IRDC G028.37+00.07. This IRDC (which we will also refer to as Cloud C, BT09) is located at a $5 \mathrm{kpc}$ distance and is one of the most massive IRDCs known with $\sim 7 \times 10^{4} M_{\odot}$ within an effective radius of about 8 pc (BTK14) (i.e., the dashed ellipse shown in Figure 1). It hosts a modest level of star formation (e.g., the MIR-bright sources in Figure 1; see also Zhang et al. 2009), but overall the star formation activity appears to be relatively low, and so this cloud should represent an earlier stage of massive star cluster formation.

The main objective and plan of the paper are as follows. The SIREX method adopts saturation-based MIREX (BT12) and FIREX (LT14) methods to then allow investigation of the extinction law variation as a function of mass surface density.
In Section 2 we review and update the results of LT14 for extinction law variation in different mass surface density regions, including utilization of WISE $12 \mu \mathrm{m}$ data. We introduce the detailed methods of SIREX mapping in Section 3. In Section 4, we present our results, first demonstrating the measurement of the Galactic background and foreground from the diffuse ISM, and then presenting the extinction law in different mass surface density environments. We discuss the implications of these results and conclude in Section 5.

\section{PHOTOMETRIC ANALYSIS}

Here we update the photometric MIREX and FIREX analysis methods of LT14 as applied to IRDC C, including: utilizing the improved $8 \mu \mathrm{m}$ extinction map of BTK14, which probes to higher values of $\Sigma$; comparing to the Draine \& $\mathrm{Li}$ (2007; hereafter DL07) diffuse ISM dust emission model; analyzing WISE $12 \mu \mathrm{m}$ data; and comparing to $\mathrm{O} 11 \mathrm{dust}$ opacity models, in addition to those of OH94.

\subsection{Dust Model Opacities in MIR to FIR Photometric Bands}

Following the methods of LT14, we consider several different dust models from $\mathrm{OH} 94$ and now also O11. The thin \& thick ice mantle models of $\mathrm{OH} 94$ (ice composition of $\mathrm{H}_{2} \mathrm{O}$ : $\mathrm{CH}_{3} \mathrm{OH}: \mathrm{CO}: \mathrm{NH}_{3}=100: 10: 1: 1$ ) and the models of $\mathrm{O} 11$ (ice composition of $\left.\mathrm{H}_{2} \mathrm{O}: \mathrm{CH}_{3} \mathrm{OH}: \mathrm{CO}: \mathrm{NH}_{3}=100: 66: 5: 5\right)$ are moderately coagulated grains (i.e., coagulation for various periods at $n_{\mathrm{H}}=10^{6} \mathrm{~cm}^{-3}$ for $\mathrm{OH} 94$ and at $n_{\mathrm{H}}=10^{5} \mathrm{~cm}^{-3}$ for $\mathrm{O} 11$. For consistency with LT14, we utilized $10^{5}$ years of coagulation OH94 models (which is equivalent to $10^{6}$ years of coagulation at $\left.n_{\mathrm{H}}=10^{5} \mathrm{~cm}^{-3}\right)$. We also adopt the gas to (refractory component) dust mass ratio of 142 (Draine 2011) that is used in LT14.

Densities of $n_{\mathrm{H}} \sim 10^{5} \mathrm{~cm}^{-3}$ are typical of dense cores and clumps within IRDCs (e.g., Tan et al. 2014); however, the lifetimes of these structures are less well constrained. We expect the clouds to be at least one local free-fall time old, i.e., $1.4 \times 10^{5}$ years at this density, but ages that are $\sim 10 \times$ longer are certainly a possibility and may help account for the high levels of deuteration that are seen in some IRDCs (Kong et al. 2015). The longest duration O11 model involves coagulation for $10^{7}$ years. The applicability of such timescales to dense clumps and cores seems less likely to be valid, especially given the apparent turbulent nature of these molecular clouds, which should induce density fluctuations on shorter timescales. We note that the $\mathrm{OH} 94$ and $\mathrm{O} 11$ are local models, valid for particular, uniform densities (c.f., the structured core models of Weidenschilling \& Ruzmaikina 1994). We note also that the OH94 and $\mathrm{O} 11$ models involve dust grain coagulation that is mediated by relative grain velocities that are set by a model of subsonic turbulence. On the larger clump scales within IRDCs, we expect supersonic turbulent motions to be more relevant, although these may be moderated by the presence of strong magnetic fields (Pillai et al. 2015). Supersonic turbulence is seen to decay within a few free-fall times in numerical simulations (Mac Low et al. 1998; Stone et al. 1998, see a review by McKee \& Ostriker 2007), so on the smaller scales of dense cores we may expect conditions to have evolved closer to a subsonic turbulent cascade.

We derive filter and background spectrum weighted opacity values. BT09 derived these for IRAC bands $1-4$ and the MIPS $24 \mu \mathrm{m}$ band using the diffuse Galactic background spectrum 

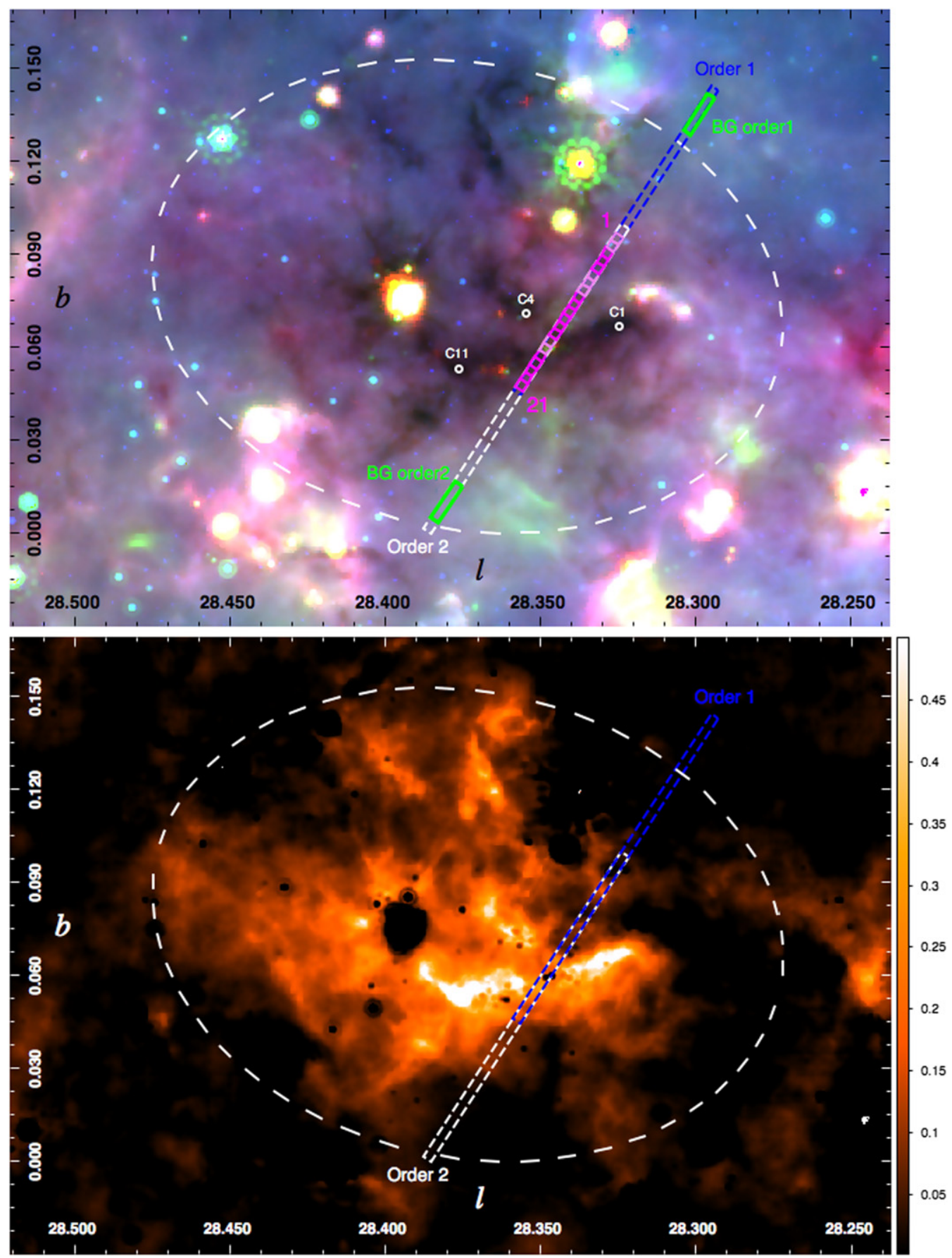

Figure 1. Upper panel: IRDC G028.37+00.07 (Cloud C) as viewed by Spitzer-IRAC $8 \mu \mathrm{m}$ (blue), Spitzer-MIPS $24 \mu \mathrm{m}$ (green), and Herschel-PACS $70 \mu \mathrm{m}$ (red). The dashed ellipse shows an approximate boundary for the IRDC defined by Simon et al. (2006). The strip observed by Spitzer-IRS is shown: Order 1 (19.5-38.0 $\mu$ m) was measured in the white rectangle region; Order $2(14.0-21.3 \mu \mathrm{m})$ in the blue rectangle. Order 1 and 2 data are both available where these regions overlap, i.e., in the magenta/violet region, which has been divided into $2110^{\prime \prime} \times 10^{\prime \prime}$ regions (numbered from upper right), from which individual spectra have been analyzed. Magenta squares indicate "dark" regions that are used for our extinction law study. Violet squares are regions we consider contaminated by $24 \mu \mathrm{m}$ point source emission. The two green regions at the outer ends of the strip are the regions used to assess the spectrum of the background (BG) emission from the diffuse ISM. Small white circles indicate the positions of the dark, saturated cores, $\mathrm{C} 1, \mathrm{C} 4, \mathrm{C} 11$, that were investigated by LT14. Bottom panel: mass surface density map (in $\mathrm{g} \mathrm{cm}^{-2}$ ) derived from Spitzer-MIPS $24 \mu \mathrm{m}$ data, $\Sigma_{24} \mu \mathrm{m}$, of the same region as the RGB map above. The slit positions of Orders 1 and 2 are shown.

from $\mathrm{Li} \&$ Draine (2001; hereafter LD01) and a total to refractory dust component mass ratio of 156 . LT14 modified these values based on the total to refractory component dust mass ratio of 142 and expanded to Herschel-PACS 70, 100, and $160 \mu \mathrm{m}$ filters. Here, we recalculate these opacities by utilizing the diffuse Galactic background emission model of DL07, and now also including the WISE $12 \mu \mathrm{m}$ band filter (Table 1).

These opacities can be used to calculate $\Sigma$ from a given set of imaging data. Their relative values will also be compared to those observed in IRDC C. 
Table 1

Telescope Band and Background-weighted Dust Opacities Per Total Mass ${ }^{\mathrm{a}}\left(\mathrm{cm}^{2} \mathrm{~g}^{-1}\right)$

\begin{tabular}{|c|c|c|c|c|c|c|c|c|c|}
\hline Dust Model ${ }^{\mathrm{b}}$ & $\begin{array}{l}\text { IRAC3.5 } \\
3.52 \mu \mathrm{m}^{\mathrm{c}}\end{array}$ & $\begin{array}{c}\text { IRAC4.5 } \\
4.49 \mu \mathrm{m}\end{array}$ & $\begin{array}{c}\text { IRAC6 } \\
5.91 \mu \mathrm{m}\end{array}$ & $\begin{array}{c}\text { IRAC8 } \\
7.80 \mu \mathrm{m}\end{array}$ & $\begin{array}{l}\text { WISE12 } \\
12.0 \mu \mathrm{m}\end{array}$ & $\begin{array}{l}\text { MIPS } 24 \\
23.0 \mu \mathrm{m}\end{array}$ & $\begin{array}{l}\text { PACS70 } \\
74.0 \mu \mathrm{m}\end{array}$ & $\begin{array}{c}\text { PACS } 100 \\
103.6 \mu \mathrm{m}\end{array}$ & $\begin{array}{l}\text { PACS160 } \\
161.6 \mu \mathrm{m}\end{array}$ \\
\hline OH94 thin mantle & $26.9(19.5)$ & 14.4 (11.7) & $10.31(9.37)$ & 8.19 (8.04) & 12.8 & 6.26 & 1.14 & 0.598 & 0.287 \\
\hline OH94 thick mantle & $52.4(38.0)$ & $17.5(14.3)$ & $20.1(18.2)$ & $10.3(10.1)$ & 36.7 & 11.4 & 3.96 & 1.26 & 0.399 \\
\hline O11 $0.1 \mathrm{My}$ & $16.3(14.1)$ & $8.16(7.57)$ & 5.14 (4.97) & $7.60(7.56)$ & 9.87 & 6.59 & 1.22 & 0.590 & 0.242 \\
\hline O11 0.3My & $41.4(25.7)$ & $18.4(12.2)$ & $9.08(6.64)$ & $8.91(8.13)$ & $11.5(11.0)$ & 7.34 (7.29) & 1.25 & 0.59 & 0.24 \\
\hline O11 1My & $115(61.3)$ & $70.5(37.8)$ & $40.3(22.0)$ & $21.7(14.1)$ & $22.5(16.0)$ & $11.4(9.17)$ & $1.34(1.19)$ & $0.57(0.53)$ & $0.21(0.20)$ \\
\hline
\end{tabular}

Notes.

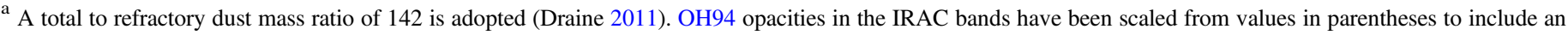

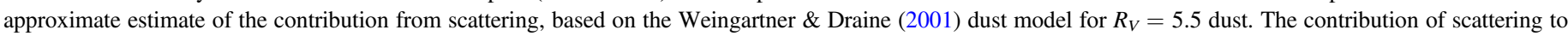

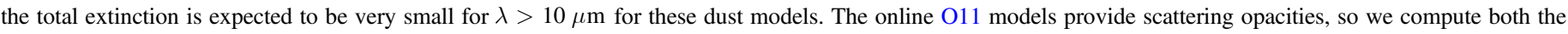
pure absorption extinction (in parentheses; shown when there is a significant difference) and total extinction including scattering.

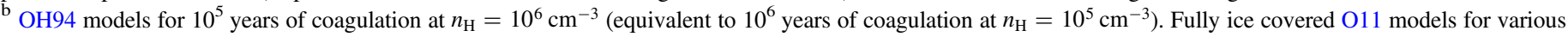
coagulation times as noted (at $n_{\mathrm{H}}=10^{5} \mathrm{~cm}^{-3}$ ).

${ }^{\mathrm{c}}$ Mean wavelengths weighted by filter response and background spectrum.

\subsection{The MIR to FIR Dust Extinction Law in IRDCs from Broadband Photometry}

We now re-visit the tentative evidence presented by LT14 of extinction law variation, i.e., flatter $\kappa_{\lambda} / \kappa_{8} \mu \mathrm{m}$, in higher $\Sigma$ regions of IRDC C. We utilize the same MIR and FIR images processed by LT14, i.e., Spitzer-IRAC 3.5, 4.5, 5.9, $8 \mu \mathrm{m}$, Spitzer-MIPS $24 \mu \mathrm{m}$, and archival data of Herschel-PACS $70 \mu \mathrm{m}$. The angular resolutions of the IRAC bands, MIPS $24 \mu \mathrm{m}$ band and PACS $70 \mu \mathrm{m}$ band are $\sim 2^{\prime \prime}, 6^{\prime \prime}$, and $6^{\prime \prime}$, respectively.

We also calibrate and use WISE $12 \mu \mathrm{m}$ imaging data with angular resolution $\sim 6 "$ "5. Following MIREX (BT09, BT12) and FIREX mapping (LT14) methods, we estimated the background emission by applying the small median filter (SMF) method, i.e., a median filter size set to $1 / 3$ of the major axis of the IRDC, i.e., $4^{\prime}$, in the region outside the dashed ellipse shown in Figure 1, followed by interpolation inside the ellipse.

We also adopted the saturation based foreground estimation method of BT12: the values of the foreground, relative to that in IRAC band 4 at $8 \mu \mathrm{m}$, were measured towards cores $\mathrm{C} 1, \mathrm{C} 4$, and $\mathrm{C} 11$ and are shown in the top panel of Figure 2. They are compared to the DL07 model of Galactic plane diffuse ISM emission (excluding stars), which is shown by the solid line, with the diamond shaped boxes showing convolution of this model with the instrumental filter response functions.

The $\Sigma_{8 \mu \mathrm{m}}$ map of IRDC C from BTK14, which probes to higher mass surface densities than the BT12 map, is now utilized so that we are able to consider binned values up to $0.4 \mathrm{~g} \mathrm{~cm}^{-2}$, compared with the $0.3 \mathrm{~g} \mathrm{~cm}^{-2}$ of LT14. Following LT14, we derive optical depth maps in $1^{\prime}$ regions around each saturated core $\mathrm{C} 1, \mathrm{C} 4$, and $\mathrm{C} 11$ in each waveband and then maps of relative opacity, normalized to that of IRAC band 4 at $8 \mu \mathrm{m}$, and with angular resolution of $\sim 6^{\prime \prime}$, set by the PACS $70 \mu \mathrm{m}$ image. We also consider opacities normalized to those derived from MIPS images at $24 \mu \mathrm{m}$.

Note that this analysis, following BT12 and LT14, assumes that the IRDC itself is a negligible source of emission, either intrinsically (e.g., from thermal emission of surface layers of warm dust, including transiently heated small grains) or from scattering of radiation into our line of sight from other directions by dust within the IRDC. This assumption appears to be supported by the fact that independent dark saturated regions are seen within the IRDC across the studied MIR to FIR wavebands. If scattering has an effect, it is expected to be most important at the shorter, MIR wavelengths, where it has been seen to occur in deep IRAC 3.5 and $4.5 \mu \mathrm{m}$ images of nearby cores, producing the "core shine" phenomenon (e.g., Pagani et al. 2010; Lefèvre et al. 2014). Accurate assessment of the effects of such scattering in IRDCs requires radiative transfer modeling and assumptions about the radiation fields and 3D IRDC structure. Given our focus in this paper on dust extinction properties at longer wavelengths, especially extending from $\sim 10$ to $\sim 70 \mu \mathrm{m}$, where scattering should be of relatively minor importance for most grain models, we continue with the approximation adopted in our previous studies of neglecting the effects scattering.

Each pixel in the maps has a value of $\Sigma_{8} \mu \mathrm{m}$. Scatter plots of $\kappa_{12 \mu \mathrm{m}} / \kappa_{8 \mu \mathrm{m}}, \kappa_{24 \mu \mathrm{m}} / \kappa_{8 \mu \mathrm{m}}$, and $\kappa_{70 \mu \mathrm{m}} / \kappa_{8 \mu \mathrm{m}}$ versus $\Sigma_{8 \mu \mathrm{m}}$ are shown in the top row of Figure 3. The bottom row shows the equivalent plots of $\kappa_{8 \mu \mathrm{m}} / \kappa_{24 \mu \mathrm{m}}, \kappa_{12 \mu \mathrm{m}} / \kappa_{24 \mu \mathrm{m}}$, and $\kappa_{70 \mu \mathrm{m}} / \kappa_{24 \mu \mathrm{m}}$ versus $\Sigma_{8 \mu \mathrm{m}}$. Note that with the new BTK14 $\Sigma$ map, including its NIR offset corrections, there are relatively few pixels with $\Sigma<0.1 \mathrm{~g} \mathrm{~cm}^{-2}$. Thus, we replace the low- $\Sigma$ bin of $0.1 \pm 0.05 \mathrm{~g} \mathrm{~cm}^{-2}$ used by LT14, with a bin defined by $\Sigma=0.15 \pm 0.05 \mathrm{~g} \mathrm{~cm}^{-2}$. Binned averages for $\Sigma_{8} \mu \mathrm{m}=$ $0.15 \pm 0.05,0.2 \pm 0.05,0.3 \pm 0.05,0.4 \pm 0.05 \mathrm{~g} \mathrm{~cm}^{-2}$ are shown - these are values of $\Sigma$ that should not be affected by saturation in the BTK14 map (these values are also shown in the third and fourth panels of Figure 2). Higher values of binned $\Sigma_{8 \mu \mathrm{m}}$ are shown in Figure 3, but these results should be treated with caution since they may be affected by saturation, especially affecting both 8 and $24 \mu \mathrm{m}$ results.

Comparing to the models of OH94, the observed $\kappa_{70 \mu \mathrm{m}} / \kappa_{8} \mu \mathrm{m}$ and $\kappa_{70 \mu \mathrm{m}} / \kappa_{24} \mu \mathrm{m}$ values are closer to the thick ice mantle case than the thin ice mantle case (similar to the findings of LT14). However, the $12 \mu \mathrm{m}$ WISE results do not favor the thick ice mantle model (see also Figure 2). Comparing to the O11 models, the observed $\kappa_{70 \mu \mathrm{m}} / \kappa_{8} \mu \mathrm{m}$ and $\kappa_{70 \mu \mathrm{m}} / \kappa_{24 \mu \mathrm{m}}$ values are consistent with models with coagulation times of $\gtrsim 3$ Myr. However, again the shorter wavelength results (including the IRAC bands 1 to 3; see Figure 2) tend to 


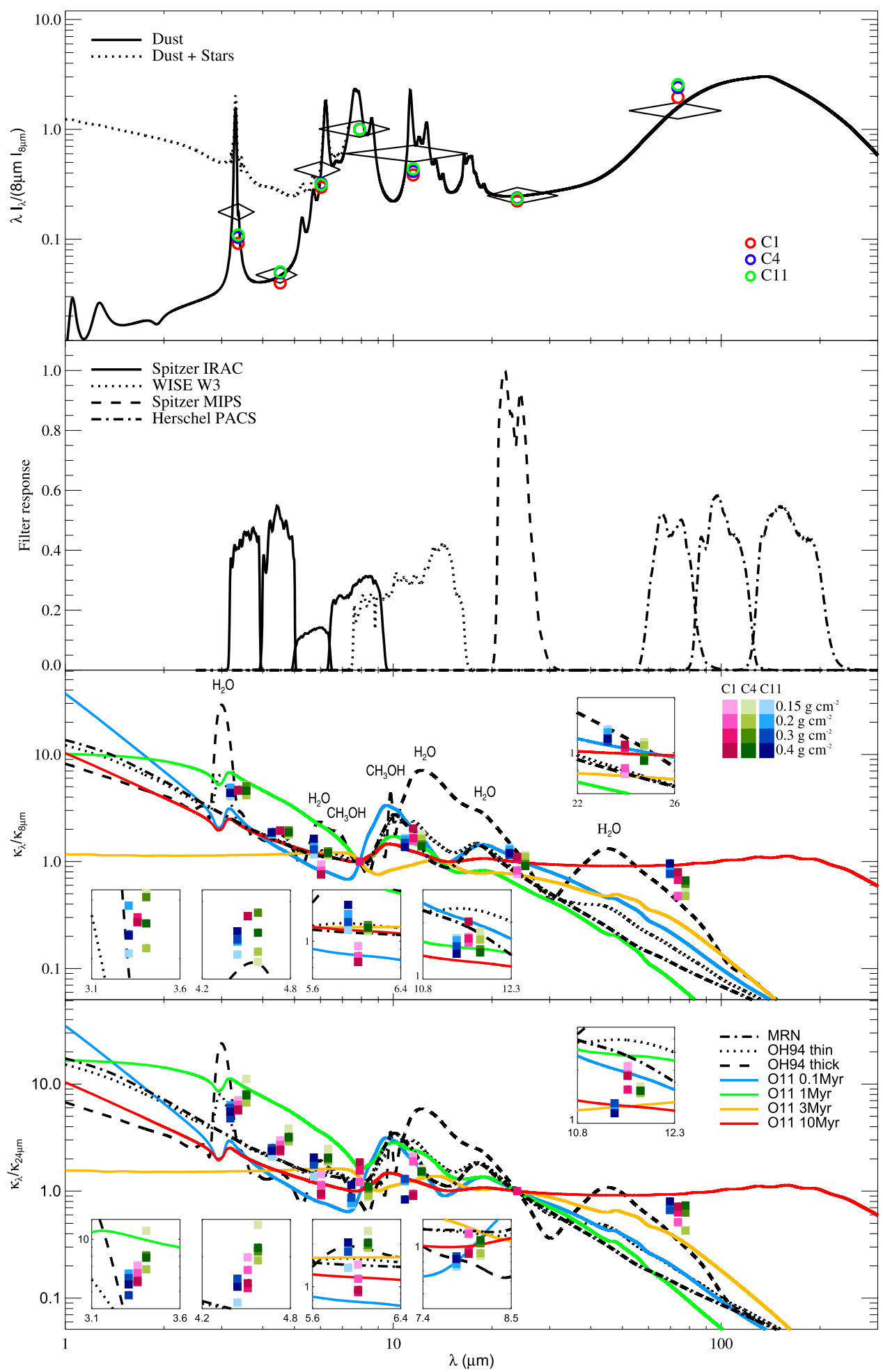

Figure 2. (a) Top: total IR to sub-mm SED of the Galactic plane diffuse ISM, normalized to the Spitzer-IRAC $8 \mu \mathrm{m}$ band flux. The solid line shows the dust-only component of the DL07 model and the dotted line indicates the total flux from dust and stellar components. Large diamonds indicate convolution of the dust-only SED with the filter response functions of corresponding instruments (panel (b)). The red, blue, and green open circles show foreground intensities, relative to the IRAC $8 \mu \mathrm{m}$ value, measured toward the three saturated cores C1, C4, and C11, respectively. (b) Second from top: filter response functions of IRAC bands 1-4 (solid lines), WISE W3 band at $\sim 12 \mu \mathrm{m}$ (dotted), MIPS $24 \mu \mathrm{m}$ (dashed) and PACS 70, 100 and $160 \mu \mathrm{m}$ (dotted-dashed). (c) Third from top: opacities relative to effective opacity measured in the IRAC $8 \mu \mathrm{m}$ band. Black dotted/dashed/dotted-dashed lines show thin/thick ice mantle and bare grain dust models of OH94 after $10^{5}$ years of coagulation at $n_{\mathrm{H}}=10^{6} \mathrm{~cm}^{-3}$ (or $10^{6}$ years of coagulation at $n_{\mathrm{H}}=10^{5} \mathrm{~cm}^{-3}$ ). Blue, green, gold, and red solid lines indicate O11 fully ice coated dust models after $0.1,1,3$ and $10 \mathrm{Myr}$ of coagulation at $n_{\mathrm{H}}=10^{5} \mathrm{~cm}^{-3}$, respectively. The red/green/blue filled squares (for $\mathrm{C} 1 / \mathrm{C} 4 / \mathrm{C} 11$ regions; slight wavelength offsets applied for clarity; see also zoom-in insets) show $\kappa_{\nu} / \kappa_{8} \mu \mathrm{m}$ for the indicated $\Sigma_{8 \mu \mathrm{m}}$ ranges. For uncertainties in these data, see Figure 3. (d) Bottom: same as (c), but now showing opacities relative to effective opacity measured in the MIPS $24 \mu \mathrm{m}$ band. 

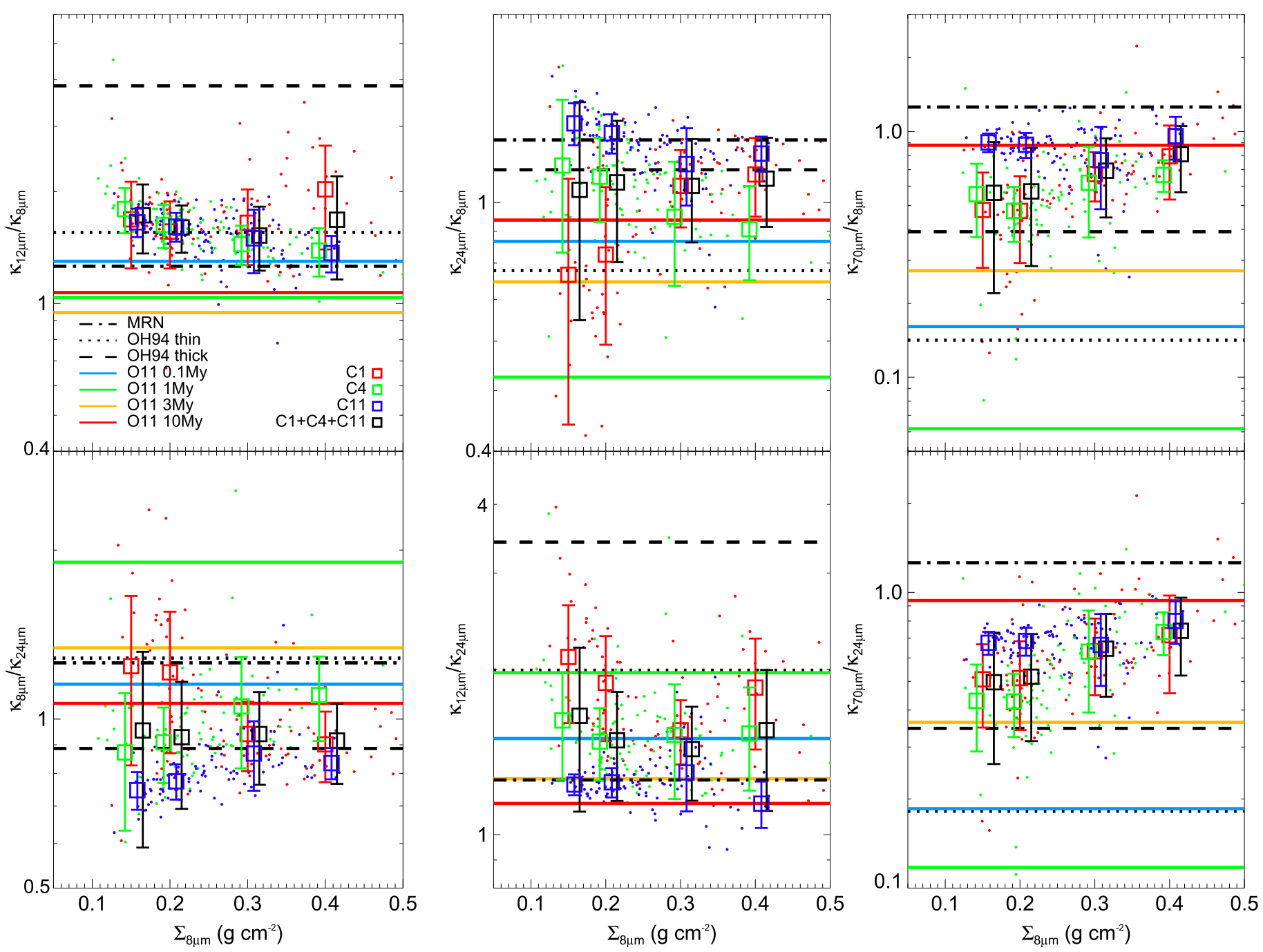

Figure 3. Scatter plots of $\kappa_{12 \mu \mathrm{m}} / \kappa_{8} \mu \mathrm{m}, \kappa_{24} \mu \mathrm{m} / \kappa_{8} \mu \mathrm{m}, \kappa_{70} \mu \mathrm{m} / \kappa_{8} \mu \mathrm{m}, \kappa_{8} \mu \mathrm{m} / \kappa_{24} \mu \mathrm{m}, \kappa_{12 \mu \mathrm{m}} / \kappa_{24} \mu \mathrm{m}$ and $\kappa_{70} \mu \mathrm{m} / \kappa_{24} \mu \mathrm{m}$ vs. $\Sigma_{8} \mu \mathrm{m}$, as measured from the BTK14 $\Sigma_{8} \mu \mathrm{m}$ map and imaging data from Spitzer-IRAC $8 \mu \mathrm{m}$, WISE $12 \mu \mathrm{m}$, Spitzer-MIPS $24 \mu \mathrm{m}$, and Herschel-PACS $70 \mu \mathrm{m}$. The size of pixels in all maps have been regridded to $\sim 6^{\prime \prime}$ resolution (i.e., the approximate beam sizes of the 12, 24, and $70 \mu \mathrm{m}$ images). Red, green, and blue dots correspond to pixels in C1, C4, and C11 regions, respectively. The open squares are the mean values of $\log _{10}$ of relative $\kappa$, with error bars indicating the $1 \sigma$ dispersion. The black open squares are the overall average of the three cores. Black dotted, dashed, and dotted-dashed lines are relative $\kappa$ values of $\mathrm{OH} 94$ thin, thick ice mantle, and bare grain models, respectively. Blue, green, gold, and red solid lines indicate $\mathrm{O} 11$ fully ice coated models of $0.1,1,3$, and $10 \mathrm{Myr}$ of coagulation time, respectively.

favor the shorter coagulation time models. In other words, there is not a single dust evolution model that provides a fully consistent fit to the data.

We next examine whether there is any trend of evolving opacity law with $\Sigma$. Inspecting the panels of Figure 3, we notice that the $\kappa_{70 \mu \mathrm{m}} / \kappa_{8} \mu \mathrm{m}$ and $\kappa_{70 \mu \mathrm{m}} / \kappa_{24} \mu \mathrm{m}$ values appear to increase as $\Sigma$ increases. We examine the Pearson correlation coefficients, $r$, and $p$-values (probability of chance correlation) for these cases finding $r=0.25, \quad 0.34, \quad$ and $p=5 \times 10^{-5}, 1 \times 10^{-8}$ when analyzing all the data for $\kappa_{70 \mu \mathrm{m}} / \kappa_{8 \mu \mathrm{m}}$, and $\kappa_{70 \mu \mathrm{m}} / \kappa_{24 \mu \mathrm{m}}$, respectively. These results appear to indicate evidence for a systematically evolving MIR to FIR extinction law, i.e., becoming flatter, as mass surface densities (and presumably also volume densities) increase. Such a flattening is expected from models of grain growth (e.g., the $\mathrm{O} 11$ models). The other wavelength data do not show trends with $\Sigma$ that are as significant. However, this may be expected since, e.g., the $\mathrm{O} 11$ models also do not predict as significant a variation as they do in comparison with the evolution of $\kappa_{70 \mu \mathrm{m}}$.
We conclude that the $70 \mu \mathrm{m}$ data in comparison with the 8 and $24 \mu \mathrm{m}$ data show evidence for growth of dust grains via coagulation (O11) and/or thickening of ice mantles (OH94, although such models struggle to match shorter wavelength results) in the densest regions. However, the particular shape of the extinction law and its possible variation are only quite poorly constrained.

\section{SIREX MAPPING METHODS}

\subsection{MIR Spectroscopic Data of IRDC G028.37+00.07}

We analyze archival Spitzer-IRS Long-Low spectra (program: 3121, PI: Kraemer, K. E.) with spectral resolution $R \sim 57-126$ of IRDC G028.37+00.07 (Cloud C). The IRS Long-Low module consists of two long slits (1st Order and 2nd Order; hereafter LL1 and LL2) that have a size of $168^{\prime \prime} \times 10$ ". 5 each. LL1 and LL2 observe the wavelength ranges of $19.5-38.0 \mu \mathrm{m}$ and $14-21.3 \mu \mathrm{m}$, respectively. The IRS spectral data of IRDC C include 4 Long-Low observations of staring modes that cover $\sim 7^{\prime}$ in length for each order (LL1 


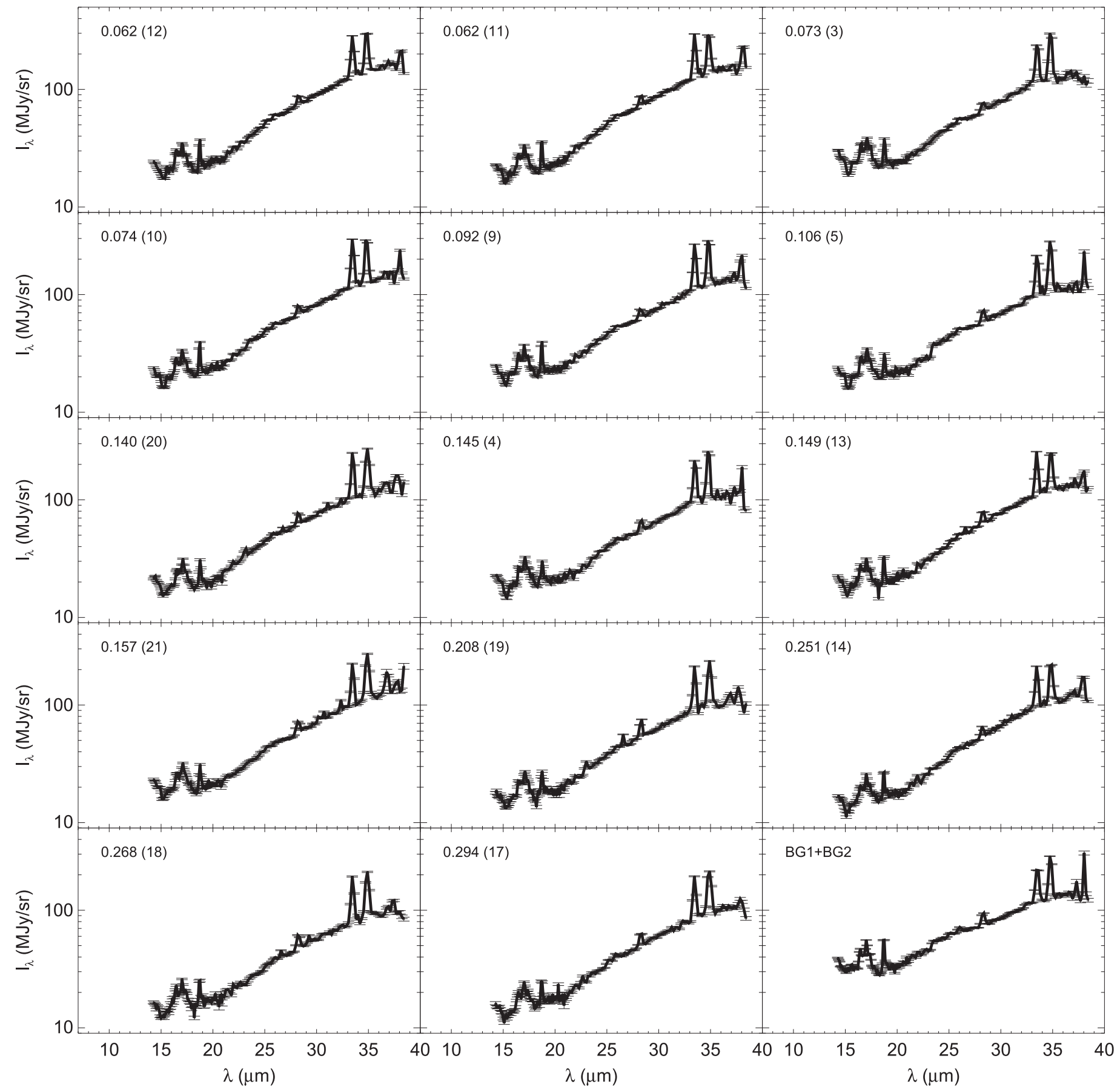

Figure 4. Extracted spectra of the selected 14 "dark" regions (i.e., without contamination of point sources) arranged in order of increasing $\Sigma_{24} \mu \mathrm{m}$ (shown in the top left corner of each panel in $\mathrm{g} \mathrm{cm}^{-2}$; the number in parentheses is the region number, see Figure 1). The summed spectrum from the background (BG) regions is shown in the lower right panel. The photometric error bars are estimated by CUBISM. All spectra are shown before the zodiacal correction has been applied.

and LL2) diagonally across the IRDC (Figure 1). The overlapped region of LL1 and LL2 is $\sim 4^{\prime}$ long. The extraction of the observed spectra is executed by utilizing the CUbe Builder for IRS Spectra Maps (CUBISM, Smith et al. 2007). The extracted spectra before any corrections are shown in Figure 4.

\subsection{Radiative Transfer Assumptions}

We follow the same simple one-dimensional radiative transfer model of the MIREX (BT12) and FIREX (LT14) methods and here review the basic equations that describe the input quantities that are required for the analysis. We need to know the intensity of radiation directed toward the observer at the location just behind, $I_{\nu, 0}$, and just in front, $I_{\nu, 1}$, of the target IRDC. The infrared emission from the IRDC (both intrinsic emission and that scattered into the line of sight) is assumed to be negligible so that

$$
I_{\nu, 1}=e^{-\tau_{\nu}} I_{\nu, 0}
$$

where optical depth $\tau_{\nu}=\kappa_{\nu} \Sigma, \kappa_{\nu}$ is total (absorption + scattering) opacity at frequency $\nu$ per unit total mass and $\Sigma$ is 


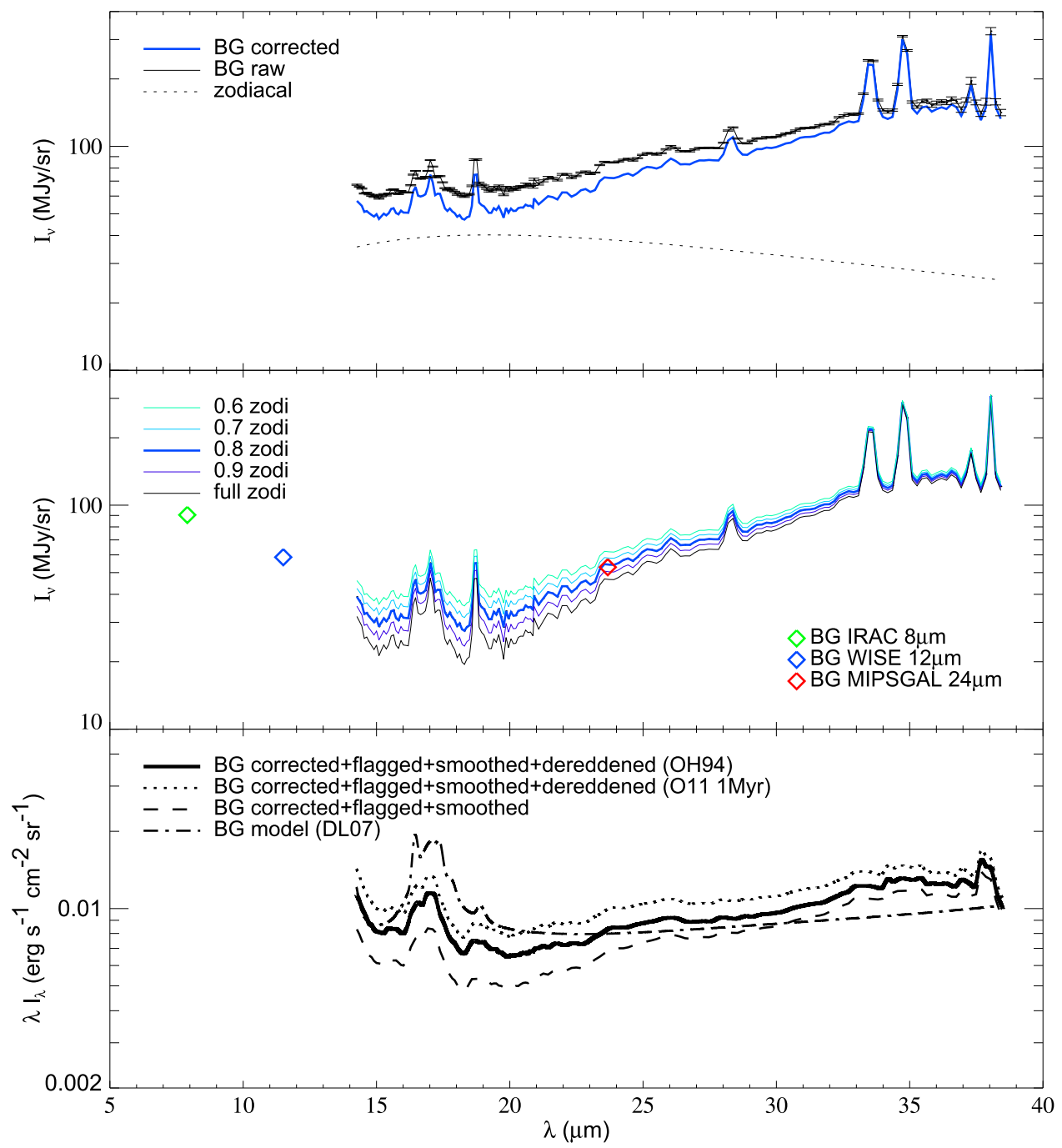

Figure 5. (a) Top: Spitzer-IRS spectrum from the background (BG) regions (green rectangles in Figure 1(a)). The black solid line shows the originally extracted spectrum without any correction (hereafter BG raw) with photometric errors. The black dotted line is a model spectrum of $\sim 0.8 \times$ the fiducial zodiacal light model (hereafter zodi). The blue solid line is the background spectrum after being corrected, i.e., BG raw- $0.785 \times$ zodi. (b) Middle: BG region spectra showing the effect of different fractions of zodiacal light subtraction as a correction to the background spectrum. The constraint of matching the Spitzer-MIPS $24 \mu \mathrm{m}$ filter-weighted data point, i.e., the average of the small median filter background model of LT14 of the two BG regions (red diamond) and also of 21 regions, sets our choice of using $0.785 \times$ the fiducial zodi model intensity. The green and blue diamonds indicate similar Spitzer-IRAC $8 \mu \mathrm{m}$ and WISE $12 \mu \mathrm{m}$ photometric data, respectively, for the BG regions. (c) Bottom: spectral line masked (see the text), smoothed (to $\sim 0.5 \mu \mathrm{m}$ resolution at $\sim 24 \mu \mathrm{m}$ ) and zodi-corrected background spectrum (dashed line), and then iteratively de-reddened via the OH94 thin ice mantle model (see the text) background spectrum (solid line). If the O11 1 Myr dust model is used for de-reddening the background then the result is the dotted line, i.e., showing about 10\%-20\% differences depending on wavelength. The dotted-dashed line indicates the DL07 model of the diffuse Galactic background emission.

the total mass surface density. The spectrum of $I_{\nu, 0}$, i.e., the background, is to be estimated from calibrated spectra of "offpositions" away from the target IRDC region, while $I_{\nu, 1}$ is derived from the observed spectrum toward the location of interest.

However, we cannot observe $I_{\nu, 0}$ and $I_{\nu, 1}$ directly because these intensities are contaminated by foreground emission, $I_{\nu \text {,fore }}$ so that the observed background emission, $I_{\nu, 0, \text { obs }}$, and the observed intensity towards the cloud, $I_{\nu, 1, \mathrm{obs}}$, are

$$
I_{\nu, 0, \mathrm{obs}}=I_{\nu, 0}+I_{\nu, \text { fore }}
$$

and

$$
I_{\nu, 1, \mathrm{obs}}=I_{\nu, 1}+I_{\nu, \text { fore }}
$$

respectively. Here we are assuming that $I_{\nu \text {,fore }}$, which is emitted from the diffuse ISM, does not vary significantly in its intensity over the face of the IRDC and to the off-position.

Therefore, as with MIREX and FIREX mapping, SIREX mapping also requires knowing the intensity of the foreground emission toward the target region. While the MIREX and FIREX derived $I_{\nu \text {,fore }}$ from observed "saturated" regions, i.e., they have same observed intensity that agrees with the minimum intensity within a range set by $2 \sigma$ (or other set range) noise level of the images, SIREX mapping toward IRDC C cannot apply this same saturation-based foreground method, since there is no slit position that is coincident with a saturated core, i.e., as defined from the MIPS $24 \mu \mathrm{m}$ maps (LT14) (see Figure 1). Thus we need to apply another method to estimate the foreground spectrum. 


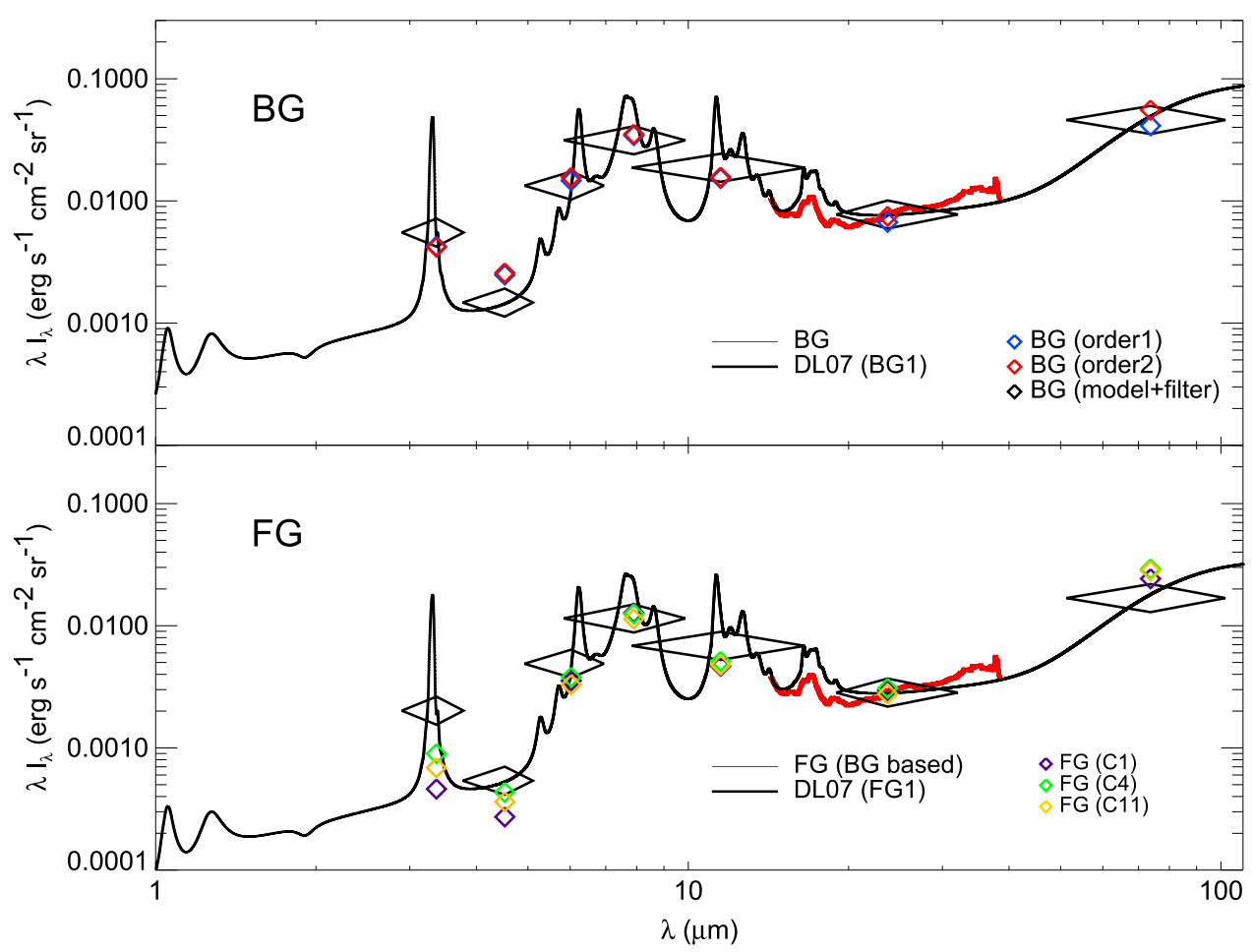

Figure 6. SED of the diffuse Galactic ISM. (a) Top: derived SED of the background emission, $I_{\nu, 0}$, from the BG regions. The red solid line shows the de-reddened and foreground-subtracted Spitzer-IRS data (see the text). The small diamonds show photometric estimates from the BG regions (based on SMF background models). The black solid line shows the DL07 model (dust only; best-fit normalized to the Spitzer-IRS data) and the large diamonds show its convolution with the filter response functions of the Spitzer-IRAC bands, WISE $12 \mu \mathrm{m}$, Spitzer-MIPS $24 \mu \mathrm{m}$, and Herschel-PACS $70 \mu \mathrm{m}$ bands. (b) Bottom: as for (a) but now showing derived SED of the foreground emission towards IRDC C. Now the small diamonds show photometric measurements towards the three saturated cores $\mathrm{C} 1, \mathrm{C} 4$ and $\mathrm{C} 11$.

\subsection{Background and Foreground Estimation}

We select two background regions, i.e., off-positions, from near the north (BG1) and south (BG2) ends of the slit position $\left(\sim 50^{\prime \prime} \times 10^{\prime \prime}\right.$ on each side). Unfortunately, these regions are not covered by both orders (Figure 1). The selected background regions have a $\sim 12 \%$ difference in their average Spitzer-MIPS $24 \mu \mathrm{m}$ photometric intensities, $\lesssim 5 \%$ differences in SpitzerIRAC $8 \mu \mathrm{m}, \sim 10 \%$ differences WISE $12 \mu \mathrm{m}$ intensities and $\sim 35 \%$ differences Herschel-PACS $70 \mu \mathrm{m}$. These gradients are utilized, below, for linear interpolation of the background spectrum along the slit.

We compare the Spitzer-IRS spectroscopic data with the Spitzer-MIPS $24 \mu \mathrm{m}$ image of the background and selected 21 regions. We subtract fiducial model zodiacal emission from the extracted spectra to obtain intrinsic intensities as a function of wavelength. However, we find that the intensities of the MIPS $24 \mu \mathrm{m}$ filter-weighted IRS background spectrum after these zodiacal light corrections have $\sim 10 \%$ higher values than in the MIPSGAL image. In order to correct this offset, we consider different fractions of the fiducial model zodiacal light subtraction from the spectra of the background and the 21 selected regions (Figure 5, middle panel). We find that the average value of the best matched fraction of the zodiacal model from all regions is $78.5 \%$.

While the BG1 and BG2 regions are near the ends of the IRS long-slit aperture, they are still quite close to the IRDC and are thus likely to be affected by extinction from the IRDC and its surrounding giant molecular cloud. Thus we de-redden the BG spectra to obtain the intrinsic background spectrum that is not contaminated by the IRDC. We first flag and mask the molecular emission lines (S(III): $18.713 \mu \mathrm{m}, \mathrm{H}_{2} \quad \mathrm{~S}$
(0): $28.221 \mu \mathrm{m}, \mathrm{S}(\mathrm{III}): 33.480 \mu \mathrm{m}$ and $\mathrm{Si}(\mathrm{II}): 34.815 \mu \mathrm{m}$ ) to simplify the spectrum. Then, de-reddening is applied to estimate $\quad I_{\nu, 0}, \quad$ via $\quad I_{\nu, 0}=I_{\nu, 1} e^{\tau_{\nu}}=\left(I_{\nu, 1, \mathrm{obs}}-I_{\nu, \text { fore }}\right) e^{\tau_{\nu}}$ (Figure 5(c)).

To estimate the amount of de-reddening, we adopt the $\Sigma_{24 \mu \mathrm{m}}$ map from the work of LT14 (see Figure 1) and $\kappa_{\nu}$ from the OH94 thin ice mantle model. Note that the validity of this assumption can be checked after the fact by comparing our derived BG spectrum with the DL07 (empirically calibrated) model, but, still, this is a fundamental limitation of the SIREX method when the off positions are too close to the IRDC, which ultimately introduces a systematic uncertainty in the shape of the derived IRDC extinction curves, although not affecting their relative shapes. To test the sensitivity of our results to this choice, we also try de-reddening the background emission using the $\mathrm{O} 11$ model of $1 \mathrm{Myr}$ coagulation time. This leads to a $\sim 20 \%$ higher intensity of the derived background (at $24 \mu \mathrm{m}$ ) than when using the OH94 thin ice mantle model (Figure 5).

Having estimated the background spectrum, we utilize it to estimate the foreground spectrum by scaling the background by a factor, $\simeq 0.35$, derived by considering the ratio of the average MIPSGAL $24 \mu \mathrm{m}$ intensity towards the three saturated cores $\mathrm{C} 1, \mathrm{C} 4$, and $\mathrm{C} 11$ compared with the average MIPSGAL $24 \mu \mathrm{m}$ SMF background map intensity of the BG1 and BG2 regions.

This estimated foreground is then used, iteratively, in the above de-reddening analysis, since the foreground is not extincted by the IRDC. We find that this iterative method converges quickly, yielding our final estimated background spectrum $\left(I_{\nu, 0}\right)$ from the BG regions and scaled foreground spectrum $\left(I_{\nu, \text { fore }}\right)$. These are shown in Figure 6, together with 


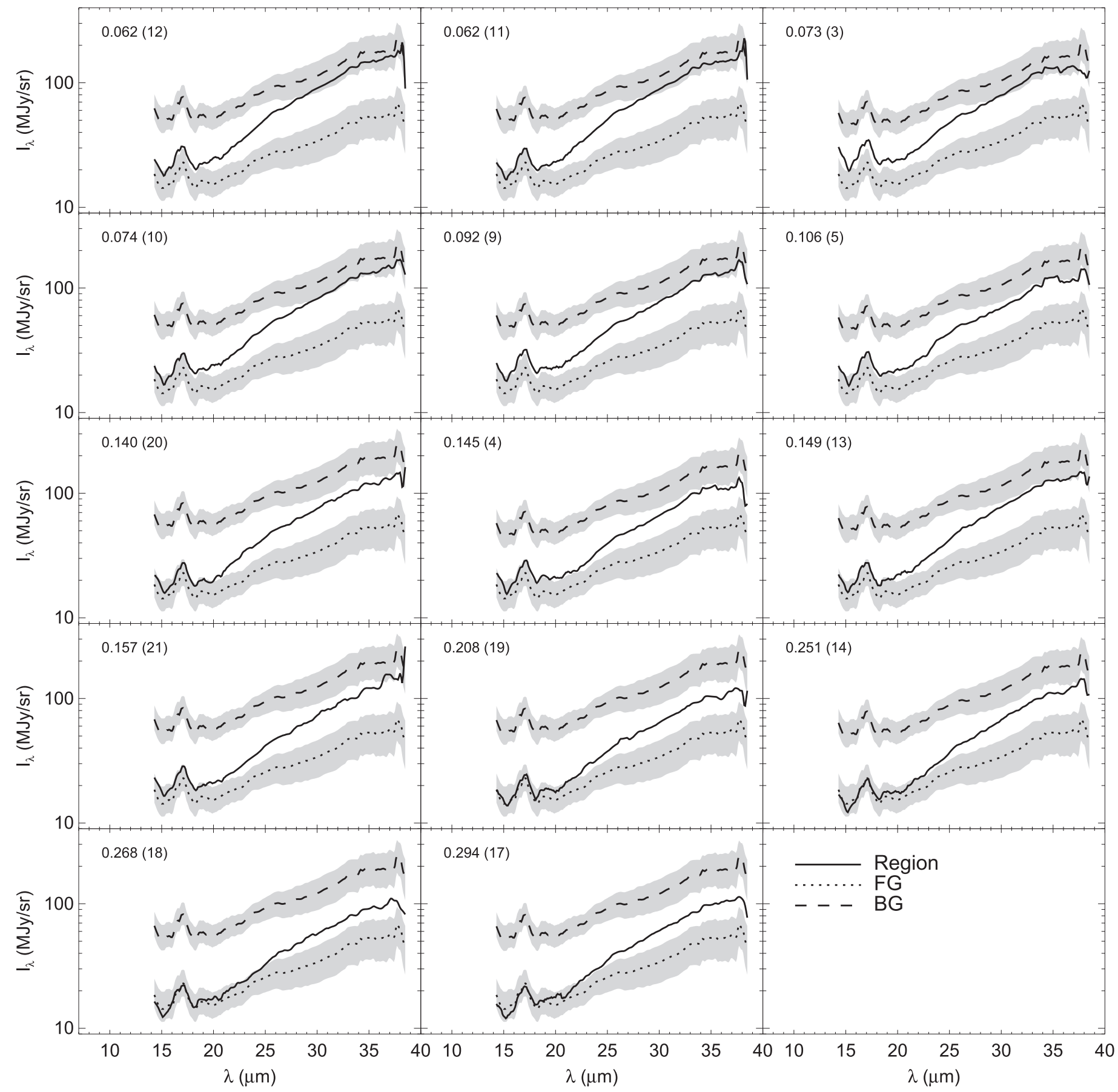

Figure 7. Derived background spectrum (dashed line; gray shaded region shows estimated errors—see text), foreground spectrum (scaled up by a factor $\sim 0.35$ from the background), and zodi-corrected observed spectra (solid lines) from each of the $1424 \mu \mathrm{m}$-dark regions (separate panels, in order of increasing $\Sigma_{24} \mu \mathrm{m}$ (indicated in $\mathrm{g} \mathrm{cm}^{-2}$ in top left corners, along with region numbers in parentheses).

our photometric estimates that probe these spectra. We also compare to the best-fitting DL07 model.

After obtaining an estimate of the background spectrum from the BG regions, we then consider how it may vary spatially along the IRS strip. To do this we utilize WISE $12 \mu \mathrm{m}$, SpitzerMIPS $24 \mu \mathrm{m}$, and Herschel-PACS $70 \mu \mathrm{m}$ photometric estimates of the background intensities (via the SMF method) at each end of the slit. Spatial gradients at these wavelengths are measured: at 12 and $24 \mu \mathrm{m}$ the intensities vary by about $10 \%$ across the length of the slit; at $70 \mu \mathrm{m}$ the variation is about $35 \%$. We use these results to carry out linear interpolation of the background intensity as a function of position and wavelength. The estimated background, i.e., $I_{\nu, 0, \text { obs }}$, is then compared to the observed emission, i.e., $I_{\nu, 1, \mathrm{obs}}$, from each of the 21 dark regions along the slit length. We find that an overall scaling of a $15 \%$ increase in the background intensity is necessary to achieve self-consistent opacity results (see Section 4). This correction is within the estimated size of 


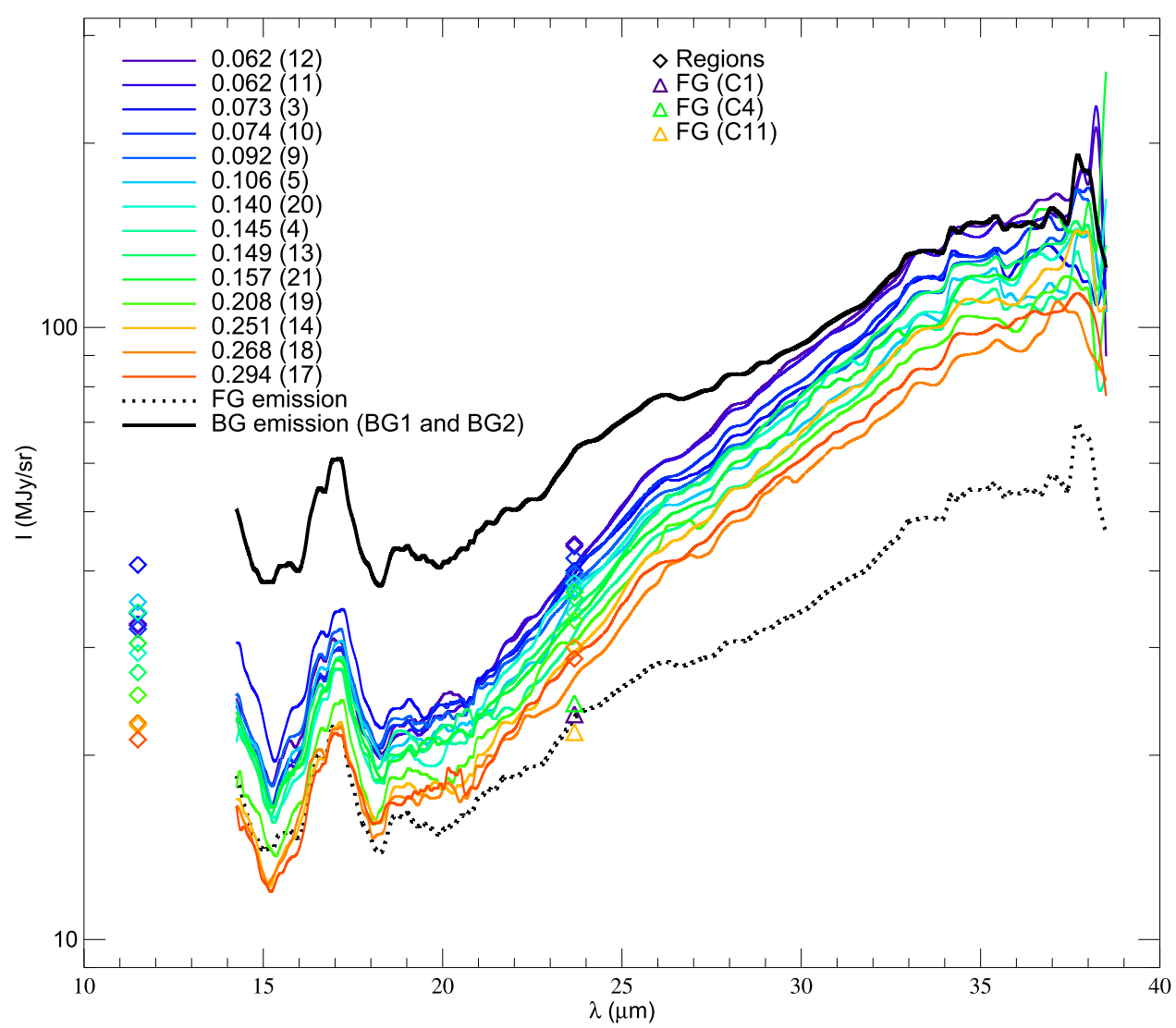

Figure 8. Extracted spectra from the $10^{\prime \prime} \times 10^{\prime \prime}$ dark regions (colored lines) and the foreground/background model (dotted/solid black lines). The comparison between the foreground spectrum and the spectra of $\Sigma_{24 \mu \mathrm{m}} \gtrsim 0.25 \mathrm{~g} \mathrm{~cm}^{-2}$ regions indicates we see saturation at $\lambda \lesssim 17 \mu \mathrm{m}$. Triangles indicate $24 \mu \mathrm{m}$ photometric data of the saturated cores C1, C4, C11 that help to normalize the foreground model (also shown in 6). Diamonds are Spitzer-MIPS $24 \mu \mathrm{m}$ photometric data points for all regions, which helped normalize the zodiacal subtraction.

systematic errors associated with the above methods, and may be due to some combination of errors in the background dereddening, background spatial interpolation, foreground fraction estimation and zodiacal correction methods. Our final adopted estimates for $I_{\nu, 0 \text { obs }}$ and $I_{\nu, 1, \text { obs }}$ are shown for each region in Figure 7.

The uncertainties of $I_{\nu, 0}$ are constrained based on the observed color variations over the 12 and $24 \mu \mathrm{m}$ maps, the observed MIPS $24 \mu \mathrm{m}$ intensity fluctuations in the surrounding regions outside the IRDC $\mathrm{C}$ ellipse, and the intrinsic photometric errors (that were shown in Figure 4). By considering the scatter in pixel by pixel values of $I_{12 \mu \mathrm{m}} / I_{24 \mu \mathrm{m}}$ in an elliptical annulus 0.7 to $1.3 \times$ the semimajor axis of the IRDC, we derive a $1 \sigma$ uncertainty, i.e., dispersion, of about $7 \%$ in the spectral slope of our estimate of the background spectrum. The color variation of the wavelength range between 24 and $38 \mu \mathrm{m}$ is uncertain and so it is potentially larger. Thus, to be conservative, we assume an uncertainty in the spectral slope in this wavelength range of $I_{\nu, 0}$ of $20 \%$. The fluctuations in $I_{24 \mu \mathrm{m}}$ are also estimated in the same annulus and found to have a $1 \sigma$ dispersion of about $18 \%$, which we adopt for our error analysis. Thus the overall uncertainties in $I_{\nu, 0}$, including photometric errors, are summed and are shown in the panels of Figure 7 as the gray shaded regions. These panels also show the scaled foreground and the zodi-corrected observed spectra for each $24 \mu \mathrm{m}$-dark region. These spectra are also shown together in Figure 8. These are the required quantities to estimate the MIR to FIR extinction law.

\section{RESULTS}

\subsection{Spectrum of the Galactic Plane Diffuse ISM}

Figure 6 shows that our estimates of the spectrum of the SED of the diffuse ISM generally agree well will the DL07 model. Our derived spectral slope of this SED from the Spitzer-IRS data rises somewhat more steeply toward longer wavelengths than the DL07 model. This could either indicate a real difference or may be due to systematic errors introduced in our analysis, such as the de-reddening method, which assumed the OH94 thin ice mantle model opacities.

As an independent check on our derived spectrum of the diffuse ISM, we can search for evidence of saturation at the short wavelength end of the observed spectra in high $\Sigma$ regions. Figures 7 and 8 show evidence for such saturation in the four highest $\Sigma$ regions (all with $\Sigma_{8 \mu \mathrm{m}}>0.2 \mathrm{~g} \mathrm{~cm}^{-2}$ ) at $\lambda \lesssim 18 \mu \mathrm{m}$. Note that the normalization of the foreground model (dotted lines in this figure) is based on the $24 \mu \mathrm{m}$ photometry towards the three saturated cores $\mathrm{C} 1, \mathrm{C} 4$, and $\mathrm{C} 11$, so if our model of emission from the diffuse ISM were in error, then we would not expect to see saturation, i.e., coincidence of the foreground model with the observed spectra.

\subsection{The MIR to FIR Extinction Law and Evidence for Grain Growth}

We calculate the optical depth, $\tau_{\nu}$, of each selected $10^{\prime \prime} \times 10^{\prime \prime} 24 \mu$ m-dark region via $\tau_{\nu}=\ln \left(I_{\nu, 0} / I_{\nu, 1}\right)$, and these 


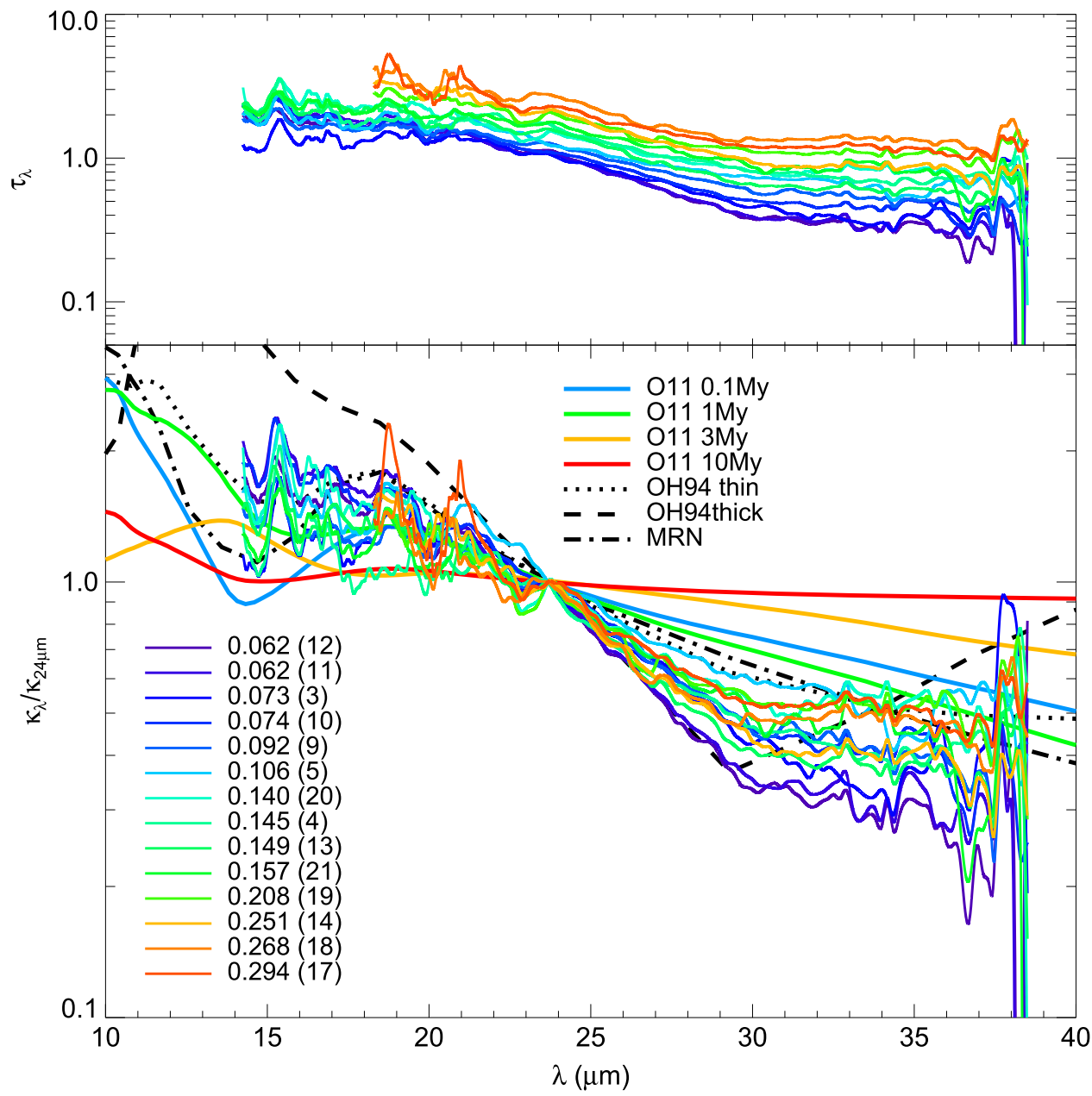

Figure 9. Upper panel: the optical depth $\left(\tau_{\lambda}\right)$ curves of the 14 selected dark positions of the Spitzer-IRS slit on the IRDC G028.37+00.07. The color of the spectra are same as in Figure 8 and are listed in the legend of the lower panel. Lower panel: relative $\kappa$ curves (normalized to $\kappa_{24} \mu \mathrm{m}$ ) of the same regions are compared to model relative opacities of OH94 thin (black dotted line) and thick ice mantle models (black dashed line), OH94 bare grain model (dotted-dashed line) and O11 models of $0.1,1,3$, and $10 \mathrm{Myr}$ of coagulation time (blue, green, gold, and red solid lines).

are shown in the upper panel of Figure 9. As expected, higher $\Sigma_{24 \mu \mathrm{m}}$ regions show higher optical depths. Using $\Sigma_{24 \mu \mathrm{m}}$ (from the LT14 FIREX map), we then derive $\kappa_{\nu}=\Sigma_{24} \mu \mathrm{m} / \tau_{\nu}$, and show $\kappa_{\lambda} / \kappa_{24 \mu \mathrm{m}}$ in the lower panel of Figure 9, along with the OH94 and O11 dust models. The absolute values of $\kappa_{\lambda}$ are shown in Figure 10.

To quantify the shape of the extinction laws that are probed by the Spitzer-IRS data we fit various spectral indices, $\beta$, where $\left.\kappa_{\lambda} / \kappa_{24 \mu \mathrm{m}} \propto \lambda^{\beta}\right)$. We define Index $\mathrm{A}$ as $\beta_{\mathrm{A}}$ being measured from 23 to $30 \mu \mathrm{m}$; Index $\mathrm{B}$ as $\beta_{\mathrm{B}}$ being measured from 30 to $34 \mu \mathrm{m}$; and Index $\mathrm{C}$ as $\beta_{\mathrm{C}}$ being measured from 23 to $34 \mu \mathrm{m}$. The divisions of $\mathrm{A}$ and $\mathrm{B}$ at $30 \mu \mathrm{m}$ were chosen to accentuate the "V"-shaped water ice feature that is prominent in the thick ice mantle models of $\mathrm{OH} 94$. Figure 11 shows these various indices and the combination of $\beta_{\mathrm{B}}-\beta_{\mathrm{A}}$ as a function of $\Sigma_{24 \mu \mathrm{m}}$. The uncertainties of the spectral indices of each region are approximately estimated by adopting the range of indices that are allowed from the upper and lower limits of the SIREXderived opacities (i.e., from the shaded areas of Figure 10).

The absolute values of these indices and the combination $\beta_{\mathrm{B}}-\beta_{\mathrm{A}}$ are consistent with the OH94 thin ice mantle model. The values of $\beta_{\mathrm{B}}$ disfavor the thick ice mantle model. O11 models with $\sim 1$ Myr of coagulation do reasonably well, but in general the O11 models are too flat. However, we caution that there are systematic errors (e.g., the dereddening of the background regions) that could be affecting the derived spectral indices of the extinction laws.

Next we look for systematic variation of the extinction laws with mass surface density. All three indices and the combination $\beta_{\mathrm{B}}-\beta_{\mathrm{A}}$ show hints of flattening as $\Sigma_{24} \mu \mathrm{m}$ increases. The Pearson $p$ values are reasonably significant for Index B and C ( $\sim 0.008$ and $\sim 0.01$, respectively). Thus Index $\mathrm{B}$ shows relatively significant changes with increasing $\Sigma_{24} \mu \mathrm{m}$, which may indicate that water ice is in fact beginning to change the shape of the extinction curves in the denser regions (although not to the extent of OH94 thick ice mantle models). Such an interpretation would help to reconcile the results of the SpitzerIRS extinction curves with the photometric results of Section 2, which we present together in a summary in Figure 12.

\section{CONCLUSIONS}

We have presented a new method of MIR to FIR spectroscopic extinction (SIREX) mapping of an IRDC, which can observationally trace grain growth processes in a region that is 


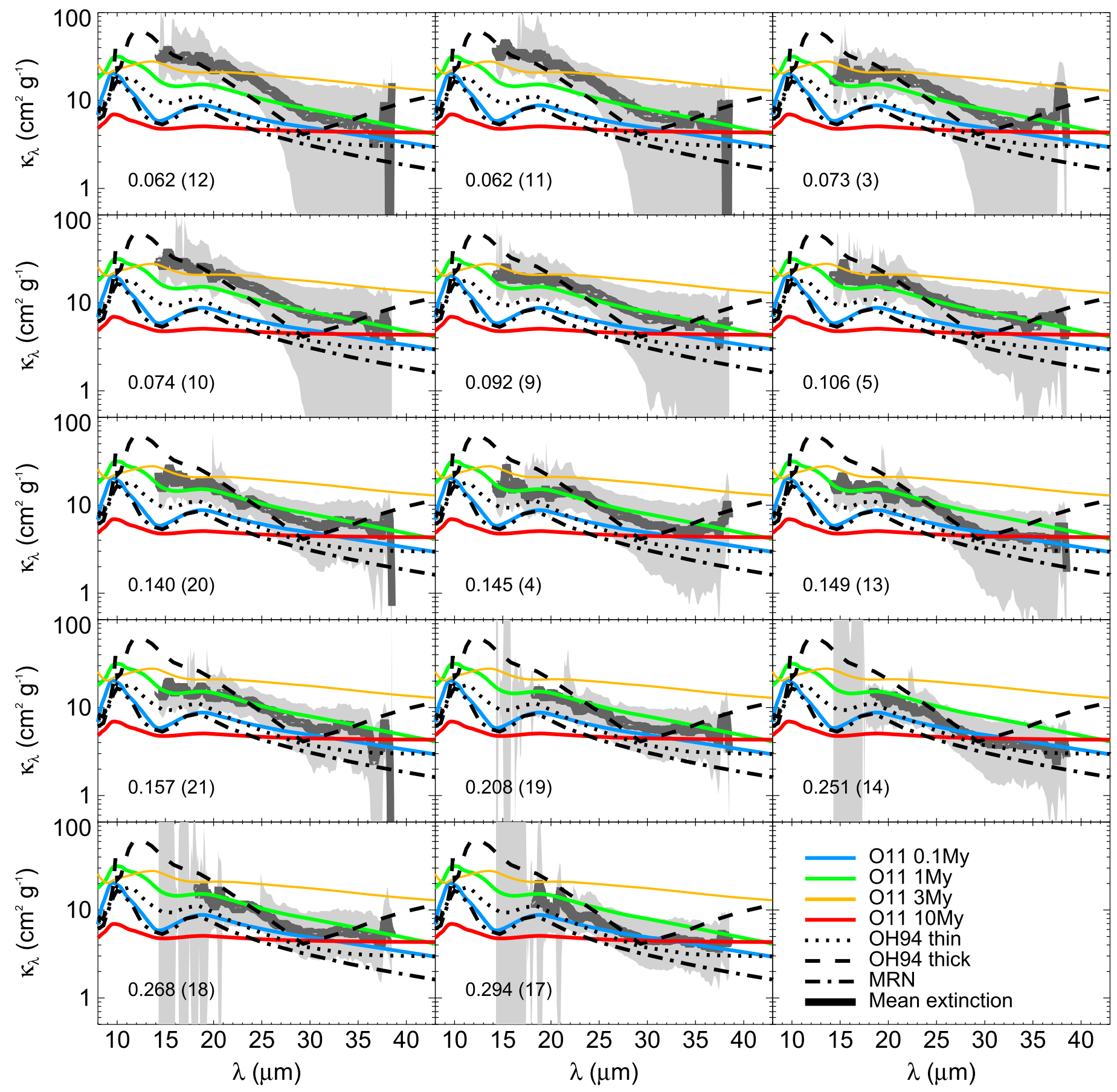

Figure 10. Opacity $\left(\kappa_{\lambda}\right)$ curves (thick solid gray line; shaded areas show uncertainties) of the individual regions (each panel lists $\Sigma_{24} \mu \mathrm{m}$ in $\mathrm{g} \mathrm{cm}^{-2}$ and region number in parentheses), compared to model $\kappa_{\lambda}$ of the OH94 thin ice mantle (dotted), thick ice mantle (dashed), and bare grain (MRN) model (dotted-dashed). The O11 models of $0.1,1,3$, and $10 \mathrm{Myr}$ of coagulation time (blue, green, gold, and red solid lines) are also shown.

expected to form massive stars and a massive star cluster. This is the first study to determine the detailed extinction law in the wavelength range $14-38 \mu \mathrm{m}$ in such dense regions. In combination with our MIREX and FIREX maps, we are also able to search for systematic variation in the extinction law as a function of mass surface density.

Our new results, especially from WISE $12 \mu \mathrm{m}$ MIREX mapping and Spitzer-IRS SIREX mapping, indicate that $\kappa_{\lambda} / \kappa_{24 \mu \mathrm{m}}$ is relatively flat from $\sim 5$ to $\sim 25 \mu \mathrm{m}$. The strong water ice absorption feature of the OH94 thick ice mantle models is not seen. SIREX results then indicate a relatively steep drop of $\kappa_{\lambda} / \kappa_{24} \mu \mathrm{m}$ out to $\sim 30 \mu \mathrm{m}$, but then hints of some flattening, especially in denser regions, out to $\sim 35 \mu \mathrm{m}$. The FIREX results at $70 \mu \mathrm{m}$ suggest this flattening extends out to these long wavelengths and is also stronger in higher density environments. Comparison with OH94 and O11 dust models 
(a)

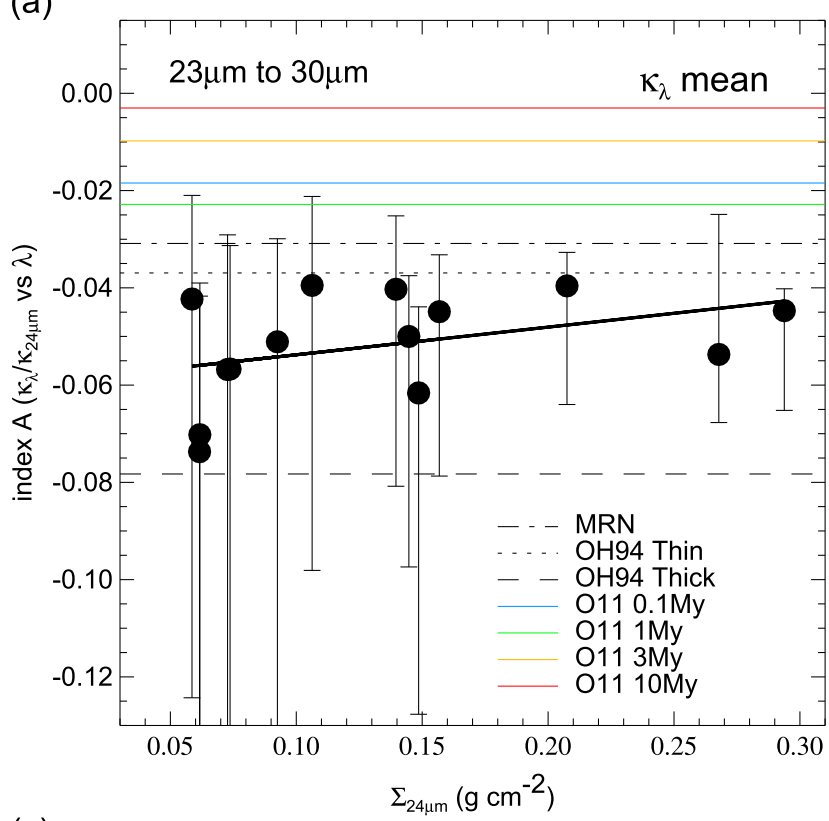

(c)

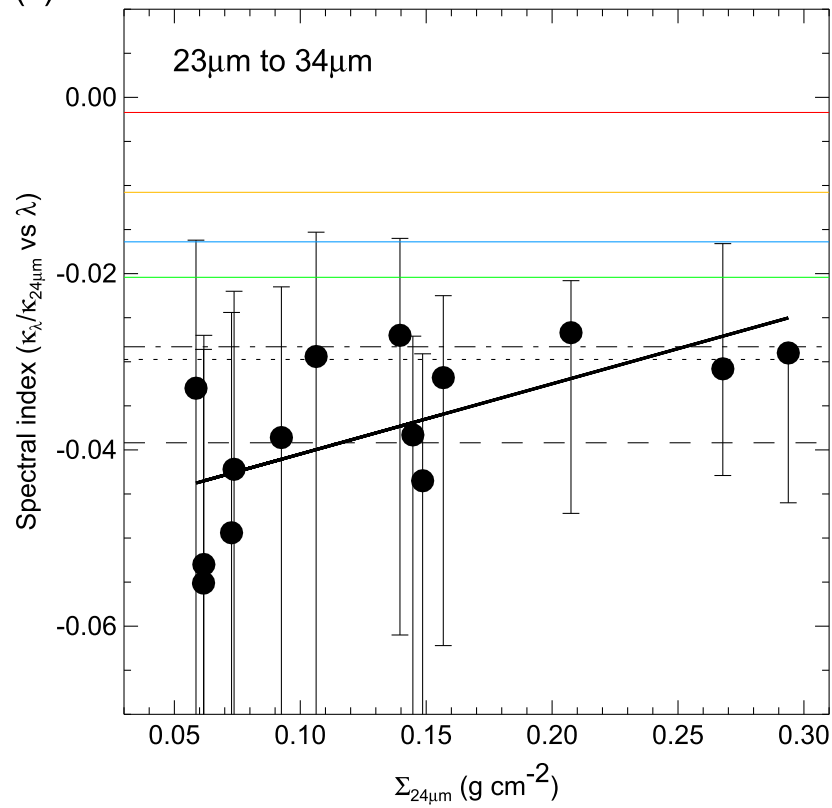

(b)

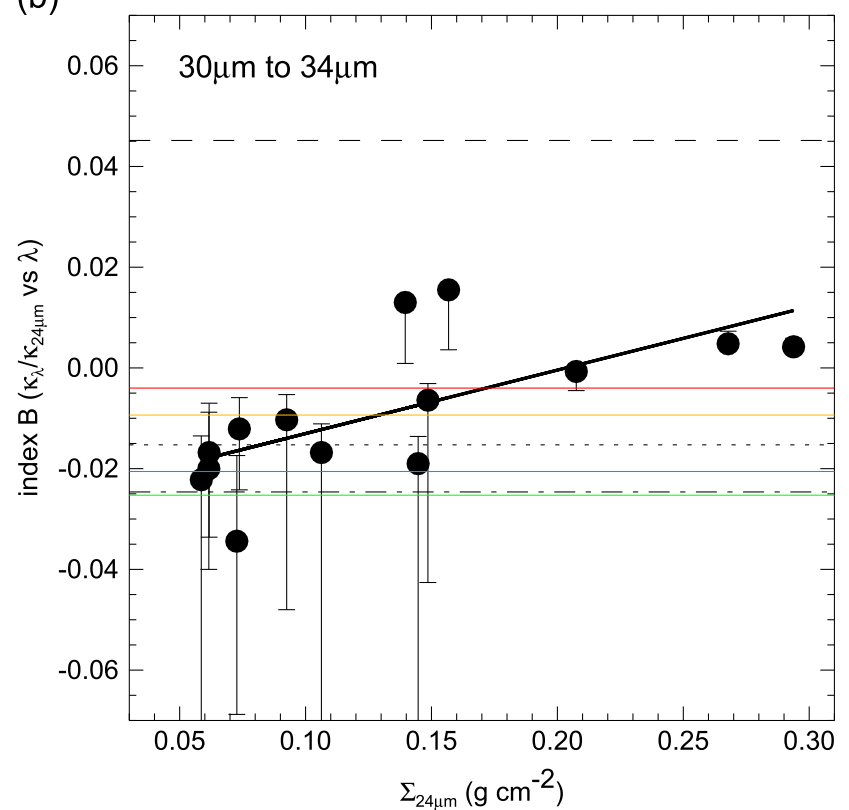

(d)

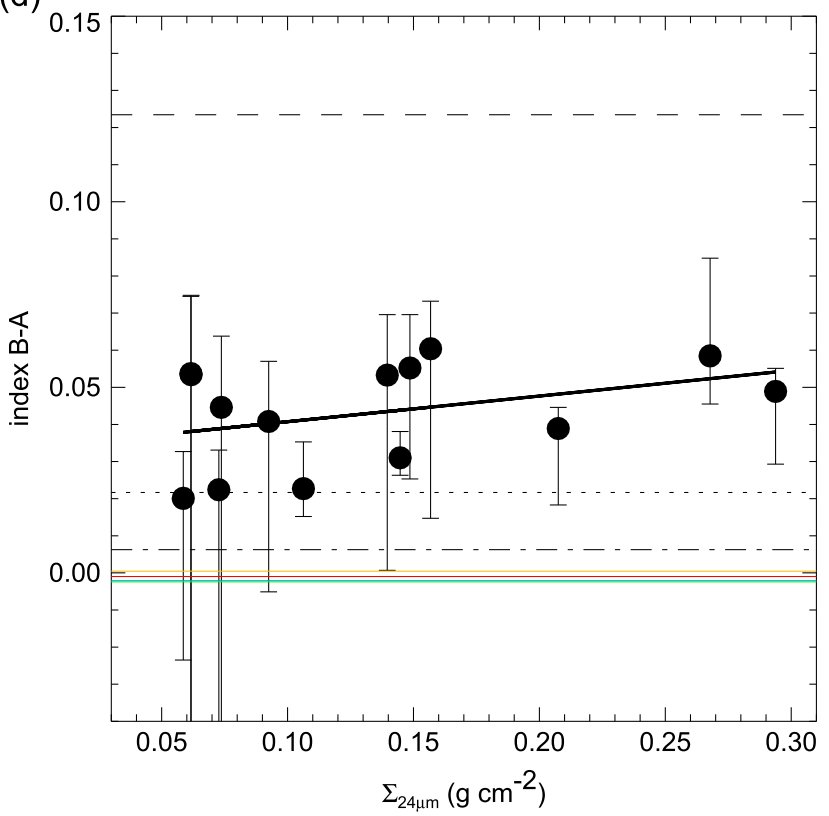

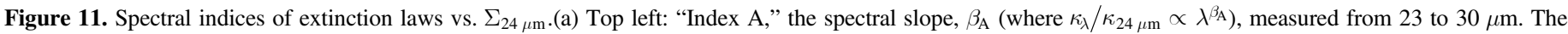

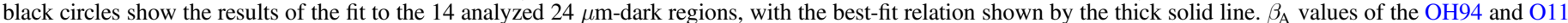

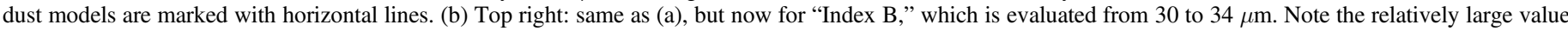

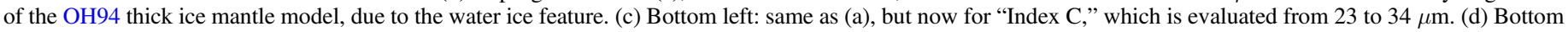
right: $\beta_{\mathrm{B}}-\beta_{\mathrm{A}}$ vs. $\Sigma_{24} \mu \mathrm{m}$.

suggests that this may require some combination of water ice mantle growth and grain coagulation.

One implication of these results is that there could be very significant grain growth on large scales within IRDCs, so that pre-stellar cores should be modeled with such large grains. This can potentially affect cooling rates (generally leading to cooler equilibrium temperatures; e.g., Keto \& Caselli 2008). It can also affect astrochemistry and ionization fractions.

Another important implication is for mass estimates of dense regions from sub-mm and $\mathrm{mm}$ dust emission. The difference in derived mass surface density that results from using either thin or thick OH94 dust opacities is about a factor of three.
Future studies of spectroscopic observation toward dense cores and clumps using finer angular resolution and higher sensitivity (e.g., JWST-MIRI) will help to better explore the evidence of grain growth in different star-forming environments.

We thank E. Schisano for WISE, Herschel data and discussions, and M. J. Butler, B. T. Draine, J. G. Ingalls, S. Kong, A. Li, and R. Paladini for discussions. The comments of an anonymous referee helped to improve the article. J.C.T. acknowledges support from NASA Astrophysics Data Analysis Program grants ADAP10-0110 and ADAP14-0135. 


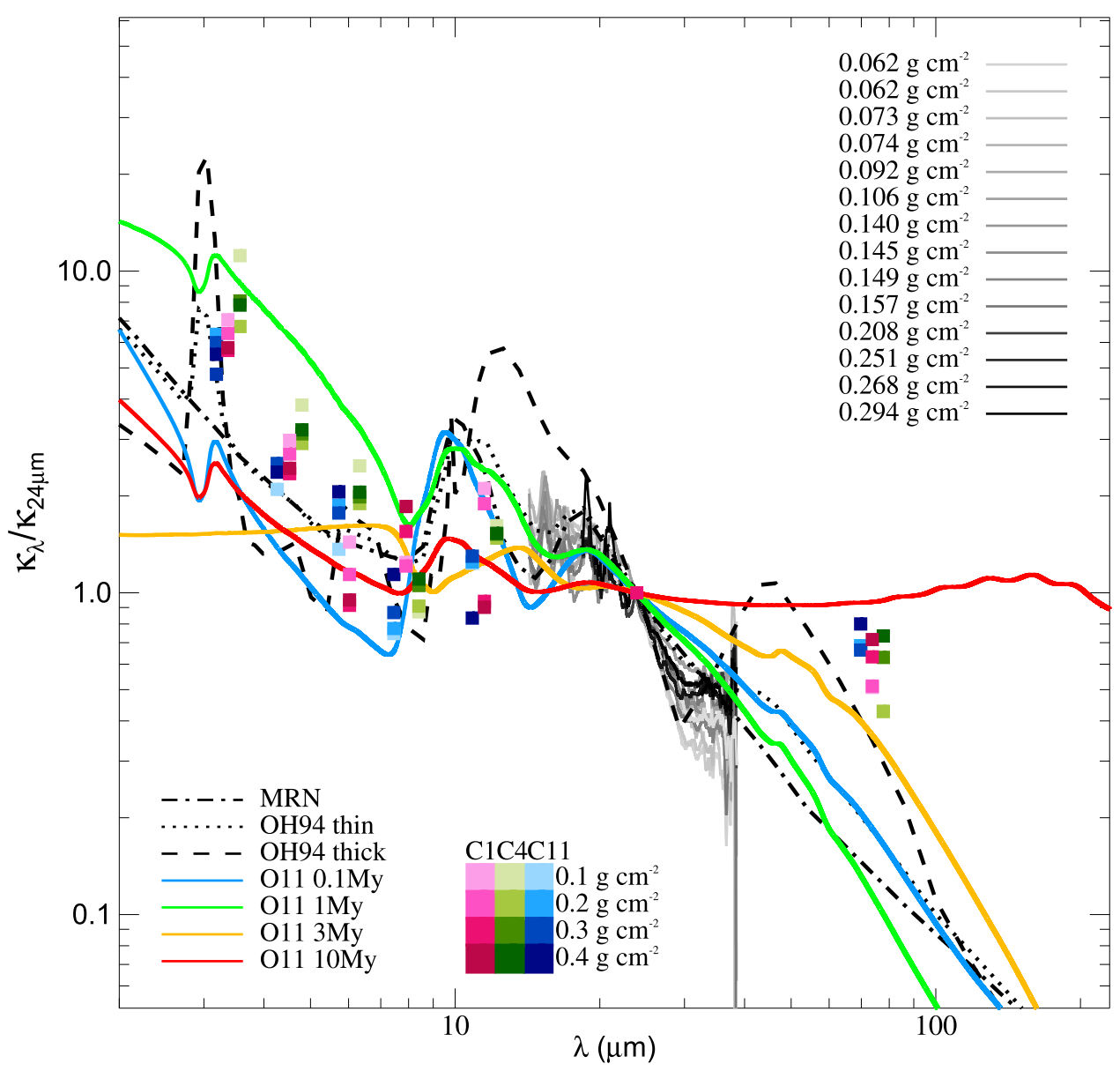

Figure 12. Overall $24 \mu \mathrm{m}$-normalized MIR to FIR extinction law, combining photometric (MIREX and FIREX) results of Section 2 (from Figure 2 (d)) and spectroscopic (SIREX) results. The solid lines with black-white gradient indicate SIREX $\kappa_{\lambda} / \kappa_{24} \mu \mathrm{m}$ results at different $\Sigma_{24} \mu \mathrm{m}$.

\section{REFERENCES}

Ascenso, J., Lada, C. J., Alves, J., et al. 2013, A\&A, 549, 135 Boogert, A. C. A., Chiar, J. E., Knez, C., et al. 2013, ApJ, 777, 73 Butler, M. J., \& Tan, J. C. 2009, ApJ, 696, 484

Butler, M. J., \& Tan, J. C. 2012, ApJ, 754, 5

Butler, M. J., Tan, J. C., \& Kainulainen, J. 2014, ApJ, 782L, 30

Chapman, N. L., Mundy, L. G., Lai, S.-P., \& Evans, N. J. 2009, ApJ, 690, 496

Cardelli, J. A., Clayton, G. C., \& Mathis, J. S. 1989, ApJ, 345, 245

Carey, S. J., Clark, F. O., Egan, M. P., et al. 1998, ApJ, 508, 721

Carey, S. J., Noriega-Crespo, A., Mizuno, D. R., et al. 2009, PASP, 121, 76

Churchwell, E., Babler, B., Meade, M., et al. 2009, PASP, 121, 213

Draine, B. T. 1989, in Infrared Spectroscopy in Astronomy, ed. B. H. Kaldeich

(ESA Special Publication, Vol. 290; Noordwijk: ESA), 93

Draine, B. T. 2011, ApJ, 732, 100

Draine, B. T., \& Li, A. 2007, ApJ, 675, 810

Flaherty, K. M., Pipher, J. L., Megeath, S. T., et al. 2007, ApJ, 663, 1069

Kainulainen, J., \& Tan, J. 2013, A\&A, 549, 53

Keto, E., \& Caselli, P. 2008, ApJ, 683, 238

Kong, S., Caselli, P., Tan, J. C., et al. 2015, ApJ, 804, 98

Lefèvre, C., Pagani, L., Juvela, M., et al. 2014, A\&A, 572, 20

Li, A., \& Draine, B. T. 2001, ApJ, 554, 778
Lim, W., \& Tan, J. C. 2014, ApJ, 780, 29

Lutz, D., Feuchtgruber, H., Genzel, R., et al. 1996, A\&A, 315, 269

Mac Low, M.-M., Klessen, R. S., Burkert, A., \& Smith, M. D. 1998, PhRvL, 80,2754

McClure, M. 2009, ApJ, 693, 81

McKee, C. F., \& Ostriker, E. C. 2007, ARA\&A, 45, 565

Ormel, C. W., Min, M., Tielens, A. G. G. M., et al. 2011, A\&A, 532, 43

Ormel, C. W., Shipman, R. F., Ossenkopf, V., \& Helmich, F. P. 2005, A\&A, 439,613

Ossenkopf, V., \& Henning, Th. 1994, A\&A, 29, 943

Pagani, L., Steinacker, J., Bacmann, A., et al. 2010, Sci, 329, 1622

Perault, M., Omont, A., Simon, G., et al. 1996, A\&A, 315, 165

Pillai, T., Kauffmann, J., Tan, J. C., et al. 2015, ApJ, 799, 74

Rathborne, J. M., Jackson, J. M., \& Chambers, E. T. 2005, ApJ, 630, 181

Simon, R., Jackson, J. M., Rathborne, J. M., \& Chambers, E. T. 2006, ApJ, 639, 227

Smith, J. D. T., Armus, L., Dale, D. A., et al. 2007, PASP, 119, 1133

Stone, J. M., Ostriker, E. C., \& Gammie, C. F. 1998, ApJ, 508, 99

Tan, J. C., Beltrán, M. T., Caselli, P., et al. 2014, PPVI, arXiv:1402.0919

Weidenschilling, S. J., \& Ruzmaikina, T. V. 1994, ApJ, 430, 713

Weingartner, J., \& Draine, B. 2001, ApJ, 563, 842

Zhang, Q., Wang, Y., Pillai, T., et al. 2009, ApJ, 696, 268 